\title{
HYPERCYCLIC ALGEBRAS FOR CONVOLUTION OPERATORS OF UNIMODULAR CONSTANT TERM
}

\author{
J. BÈS, R. ERNST, AND A. PRIETO
}

\begin{abstract}
We study the existence of hypercyclic algebras for convolution operators $\Phi(D)$ on the space of entire functions whose symbol $\Phi$ has unimodular constant term. In particular, we provide new eigenvalue criteria for the existence of densely strongly algebrable sets of hypercyclic vectors.
\end{abstract}

\section{INTRODUCTION}

Once a linear dynamical system $(X, T)$ supports a hypercyclic vector, that is, a vector $x$ whose orbit $\left\{T^{n} x\right\}_{n=0}^{\infty}$ is dense, the space $X$ always contains a dense linear subspace consisting entirely (but zero) of hypercyclic vectors 18. When we further assume $X$ to be a topological algebra, it is natural to ask whether $T$ can support a hypercyclic algebra, that is, a subalgebra of $X$ consisting entirely (but zero) of hypercyclic vectors.

Particular attention has been given to this question for the case where $X=$ $H(\mathbb{C})$ is the algebra of entire functions on the complex plane, endowed with the compact-open topology, and where $T$ is a convolution operator, that is, an operator that commutes with all translations. Godefroy and Shapiro [13] showed that such operators are precisely the ones that commute with the operator $D$ of complex differentiation, that they coincide with the operators of the form $T=\Phi(D)$ with $\Phi \in H(\mathbb{C})$ of exponential type, and that a convolution operator supports hypercyclic vectors precisely when it is not a scalar multiple of the identity.

Aron et al 2] first noted that any translation $\tau_{a}$, which is the operator $\Phi(D)$ with $\Phi(z)=e^{a z}$, fails to support a hypercyclic algebra in a dramatic way: given any $f \in H(\mathbb{C})$, the multiplicity of any zero of an element in the orbit of $f^{p}$ must be divisible by $p$. They also stopped short from giving a positive answer for $D$, showing that for the generic element $f$ of $H(\mathbb{C})$, each power $f^{n}(n \in \mathbb{N})$ is a hypercyclic vector for $D$. Shkarin [17, and independently Bayart and Matheron [4 finally showed that $D$ supports a hypercyclic algebra, prompting the question:

Question 1. Which convolution operators support a hypercyclic algebra? In other words, for which $\Phi \in H(\mathbb{C})$ of exponential type does $\Phi(D)$ support a hypercyclic algebra?

Several new positive examples were obtained since then by various authors [8, 9 , 6], including for instance when $\Phi$ is of subexponential growth having zero constant

Date: May 8th, 2019.

2010 Mathematics Subject Classification. Primary 47A16, 46 E10.

Key words and phrases. Hypercyclic algebras; convolution operators, algebrability.

This work is supported in part by MEC, Project MTM 2016-7963-P, Programme PEPS JC 2017 INSMI, and by MEC Grant MTM 2015-65825-P. 
term, or of growth order one such as $\Phi(z)=\cos (z)$ or $\Phi(z)=z e^{z}$, say. Recently Bayart [3] provided a complete characterization for the case when $\Phi(0)$ has modulus strictly smaller than one, and provided a large class of examples when $\Phi$ is of subexponential growth and has unimodular constant term:

Theorem 1.1. (Bayart) [3, Theorem 1.1] Let $\Phi$ be a non-constant entire function of exponential type.

1. Assume that $|\Phi(0)|<1$. Then the following are equivalent:

(i) $\Phi(D)$ supports a hypercyclic algebra.

(ii) $\Phi$ is not a scalar multiple of an exponential function.

2. Assume that $|\Phi(0)|=1$ and that $\Phi$ has subexponential growth. If either $\Phi^{\prime}(0) \neq 0$ or $\Phi$ has order less than $1 / 2$, then $\Phi(D)$ supports a hypercyclic algebra.

The purpose of this paper is to continue the study of hypercyclic algebras for convolution operators $\Phi(D)$ with unimodular constant term $\Phi(0)$. Notice that it suffices to understand the case when $\Phi(0)=1$, as by the León-Müller Theorem [16] any operator $T$ has the same hypercyclic vectors as a scalar multiple $\lambda T$ whenever the scalar $\lambda$ has modulus one. We can say the following (see Subsection [1.1):

Theorem 1.2. Let $\Phi$ be a non-constant entire function of exponential type, with $\Phi(0)=1$.

(a) If $\Phi$ is of subexponential growth, then $\Phi(D)$ has a hypercyclic algebra.

(b) Suppose $\Phi$ is not of subexponential growth.

1. If $\Phi$ has no zeroes, then $\Phi(D)$ has no hypercyclic algebra.

2. If $\Phi$ is of the form $\Phi(z)=e^{a z} p(z)$ for some non-constant polynomial

$$
p(z)=1+a_{1} z+a_{2} z^{2}+\cdots+a_{r} z^{r}
$$

and some non-zero scalar a with $a_{1} a^{-1} \in \mathbb{C} \backslash \mathbb{R}$ or with $2 a_{2} \neq a_{1}^{2}$, then $\Phi(D)$ supports a hypercyclic algebra.

3. If $\Phi$ has infinitely many zeros and

$$
\Phi(z)=e^{a z} \prod_{n=1}^{\infty} E_{p}\left(\frac{z}{z_{n}}\right)
$$

denotes its Hadamard factorization, we have the following whenever $\sum_{n=1}^{\infty} z_{n}^{-2} \neq 0$ :

(i) If $\sum_{n=1}^{\infty}\left|z_{n}\right|^{-1}$ converges, then $\Phi(D)$ has a hypercyclic algebra.

(ii) If $\sum_{n=1}^{\infty}\left|z_{n}\right|^{-1}$ diverges and the symbol a is non-zero then $\Phi(D)$ has a hypercyclic algebra.

The proof of Theorem 1.2 is based on Theorem 1.3 and Theorem 1.6 below.

Theorem 1.3. Let $\Phi$ be entire of exponential type with $|\Phi(0)|=1$ and

$$
\Phi^{\prime \prime}(0) \Phi(0) \neq \Phi^{\prime}(0)^{2} \text {. }
$$

Suppose that $\Phi^{-1}(\mathbb{D})$ contains arbitrarily long arithmetic progressions of the form $\{j a\}_{j=1}^{m}$, where $a \neq 0$ and $m \in \mathbb{N}$. Then $\Phi(D)$ supports a hypercyclic algebra.

Example 1.4. Let $\Phi(z)=\cos (z)$ or $\Phi(z)=\sin (z)+e^{-z}$. Then $\Phi(D)$ supports a hypercyclic algebra.

Proof. In either case we have $\Phi(0)=1$ and $\Phi^{\prime \prime}(0) \Phi(0) \neq\left(\Phi^{\prime}(0)\right)^{2}$, and $\Phi^{-1}(\mathbb{D})$ has arbitrarily long arithmetic progressions of the desired form. 
Example 1.5 below complements Case (b)3(ii) of Theorem 1.2 .

Example 1.5. Let $\Phi(z)=\frac{\sin (\pi z)}{\pi z}$. Then $\Phi(D)$ has a hypercyclic algebra.

Proof. Here $\Phi(z)=\frac{\sin (\pi z)}{\pi z}=\prod_{0 \neq k \in \mathbb{Z}} E_{1}\left(\frac{z}{k}\right)$ has zero set $\left(z_{n}\right)=(-1,1,-2,2, \ldots)$ (listed with multiplicity), which is of divergence type, and $n(r):=\#\{n \in \mathbb{N}$ : $\left.\left|z_{n}\right| \leq r\right\}$ satisfies that $\limsup _{r \rightarrow \infty} \frac{n(r)}{r}=2 \neq 0$, so $\Phi$ has order one and positive type, see [11, Theorem 2.10.3(a)]. Also $\Phi^{-1}(\mathbb{D}) \supset \mathbb{N}$, and $\Phi^{\prime \prime}(0) \Phi(0) \neq\left(\Phi^{\prime}(0)\right)^{2}$ since $\Phi(0)=1, \Phi^{\prime}(0)=0$ and $\Phi^{\prime \prime}(0)=\frac{\pi^{2}}{3}$. So $\Phi(D)$ has a hypercyclic algebra by Theorem 1.3 .

Theorem [1.6 builds on the arguments used by Bayart in [3, Theorem 3.1].

Theorem 1.6. Let $\Phi$ be of exponential type. Suppose there exist complex scalars $z_{0}, z_{1}$ with $z_{0} \in\left(0, z_{1}\right)$ so that

(i) $\quad|\Phi|<1$ on $\left(0, z_{0}\right]$ and

(ii) $\left|\Phi\left(z_{1}\right)\right|>\max \left\{1, e^{\tau_{0}\left|z_{1}\right|}\right\}$,

where

$$
\tau_{0}=\tau_{0}\left(z_{1}\right)=\limsup _{r \rightarrow \infty} \frac{\log \left|\Phi\left(r \frac{z_{1}}{\left|z_{1}\right|}\right)\right|}{r} .
$$

Then $\Phi(D)$ supports a hypercyclic algebra.

\section{Remark 1.7.}

(a) Every non-constant entire function $\Phi$ with $|\Phi(0)|=1$ satisfies condition (i) in a strong way. Indeed if $\Phi(z)=a_{0}+a_{n} z^{n}+\mathrm{o}\left(z^{n}\right)$ with $a_{n} \neq 0$ and $n \geq 1$, then there exists a subdivision of the plane into $2 \mathrm{n}$ consecutive sectors $S_{0}, \ldots, S_{2 n-1}$, each of angle $\frac{\pi}{n}$, so that for each $k=1, \ldots, n$ we have: On each ray lying in the interior of $S_{2 k-1}$ the function $\Phi$ has initially modulus strictly smaller than one, and on each ray lying in the interior of $S_{2 k}$ the function $\Phi$ initially has modulus strictly larger than one, see [12, pp 236-7].

(b) Notice that

$$
\tau_{0}=\tau_{0}\left(z_{1}\right)=\left\{\begin{array}{cl}
0 & \text { if } \rho_{z_{1}}<1 \\
h\left(\operatorname{Arg}\left(z_{1}\right)\right) & \text { if } \rho_{z_{1}}=1, \text { where }
\end{array}\right.
$$

$h=h(\theta)=\limsup _{t \rightarrow \infty} t^{-1} \log \left|\Phi\left(t e^{i \theta}\right)\right|$ is the Phragmén-Lindelöf indicator function of $\Phi$ and where $\rho_{z_{1}}$ is the growth order of $\Phi$ in the direction of $z_{1}$, that is,

$$
\rho_{z_{1}}:=\limsup _{t \rightarrow \infty} \frac{\log \log \left|\Phi\left(t \frac{z_{1}}{\left|z_{1}\right|}\right)\right|}{\log t} .
$$

In particular, $\tau_{0}\left(z_{1}\right)=0$ whenever $\Phi$ is of subexponential growth, and Theorem [1.6 may be re-stated as follows:

"Let $\Phi$ be entire of exponential type, and let $h$ be its Phragmén-

Lindelöf indicator function. Suppose there exist $\theta \in[0,2 \pi)$, and positive scalars $r, R$ so that

$$
\begin{cases}\left|\Phi\left(t e^{i \theta}\right)\right|<1 & \text { for } 0<t<r, \text { and } \\ \left|\Phi\left(R e^{i \theta}\right)\right|>\max \left\{1, e^{h(\theta) R}\right\} . & \end{cases}
$$

Then $\Phi(D)$ supports a hypercyclic algebra." 
Once a hypercyclic algebra is determined for a given operator, it is natural to ask how large such an algebra can be. While the hypercyclic algebras for $D$ obtained in [17, 4] were singly generated and thus not dense in $H(\mathbb{C})$, it was recently shown that $D$ and many other convolution operators support hypercyclic algebras that are both dense and infinitely generated [14, 10, 6]. Moreover, for the case when $|\Phi(0)|<$ 1 , the convolution operator $\Phi(D)$ supports a dense infinitely generated hypercyclic algebra if and only if it is not a scalar multiple of an exponential function, see [3, Theorem 6.3]. For the unimodular case we can say the following.

Theorem 1.8. Let $\Phi$ be a non-constant entire function of exponential type, with $|\Phi(0)|=1$. Then the generic element $f=\left(f_{n}\right)_{n=1}^{\infty}$ of $H(\mathbb{C})^{\mathbb{N}}$ freely generates a dense hypercyclic algebra for $\Phi(D)$ in each of the following cases:

(a) $\Phi$ is of subexponential growth and $\min \left\{n \in \mathbb{N}: \Phi^{(n)}(0) \neq 0\right\}$ is odd.

(b) $\Phi$ is of the form $\Phi(z)=e^{a z+b} p(z)$ for some $a, b \in \mathbb{C}$ with $a \neq 0=\operatorname{Re}(b)$ and some non-constant polynomial $p(z)=1+a_{1} z+a_{2} z^{2}+\cdots+a_{r} z^{r}$ with $a_{1} a^{-1} \in \mathbb{C} \backslash \mathbb{R}$ or with both $2 a_{2} \neq a_{1}^{2}$ and $a_{1}+a \neq 0$.

(c) $\Phi$ has Hadamard factorization $\Phi(z)=e^{a z+b} \prod_{n=1}^{\infty} E_{p}\left(\frac{z}{z_{n}}\right)$ satisfying

$$
\sum_{n=1}^{\infty} z_{n}^{-2} \neq 0, a \neq 0=\operatorname{Re}(b), \text { and } \sum_{n=1}^{\infty} z_{n}^{-1} \neq a \text { if } \sum_{n=1}^{\infty}\left|z_{n}\right|^{-1}<\infty \text {. }
$$

For a statement to hold at a generic element of an $F$-space $X$ we mean that it holds at each element of a dense $G_{\delta}$ subset of $X$.

Remark 1.9. In the language of Lineability [1], Theorem 1.8 states in particular that under each of the assumptions $(a)-(c)$ the set of hypercyclic vectors of the operator $\Phi(D)$ is densely strongly algebrable in the sense of Bartoszewicz and Głąb [5], see also [10, Section 2]: A subset $S$ of a commutative topological algebra $X$ is said to be densely strongly algebrable provided $S \cup\{0\}$ contains a dense subalgebra of $X$ that is induced by an infinite set of free generators.

The paper is organized as follows. In Section 2 we show Theorem 1.3 . In Section 3 we show Theorem 1.6 and (all but Case (b)3(ii) of) Theorem 1.2. In Section 4 we show Theorem 1.8, which require stronger versions of Theorem 1.3 and Theorem 1.6 -Theorem 4.2 and Theorem 4.4, respectively- whose proofs are necessarily more technical. A particular case of the former, Corollary 4.3, establishes the pending Case (b)3(ii) of Theorem 1.2

1.1. Notation and preliminaries. We recall that the order $\rho$ of a constant entire function is defined as zero, and for a given non-constant $f \in H(\mathbb{C})$ it is given by $\rho:=$ $\limsup _{r \rightarrow \infty} \frac{\log \log M(r)}{\log r}$, where $M(r)=M_{f}(r)=\sup _{|z| \leq r}|f(z)|$. When $f$ has finite order $\rho$, its type $\tau$ is given by $\tau:=\limsup _{r \rightarrow \infty} r^{-\rho} \log M(r)$. Following Boas [11, we say that $\Phi$ is of growth $(\rho, \tau)$ provided it is either of order less than $\rho$ or, if of order equal to $\rho$, it has type not exceeding $\tau$. We also say that $\Phi$ is of subexponential growth provided it is of growth $(1,0)$, and that $\Phi$ is of exponential type provided it is of growth $(1, \tau)$ for some $\tau<\infty$. An entire function $\Phi(z)=\sum_{n=0}^{\infty} a_{n} z^{n}$ is of exponential type precisely when there exist constants $C, R \in(1, \infty)$ so that $\left|a_{n}\right| \leq$ $C R^{n}(n \in \mathbb{N})$, and this holds precisely when the operator $\Phi(D): H(\mathbb{C}) \rightarrow H(\mathbb{C})$, $f \mapsto \sum_{n=0}^{\infty} a_{n} D^{n} f$, is well defined. 
According to the Hadamard Factorization Theorem [11, Theorem 2.7.1], each $f \in H(\mathbb{C})$ of finite order $\rho$ with zero set $\left(z_{n}\right)$ listed with multiplicity and satisfying $0<\left|z_{1}\right| \leq\left|z_{2}\right| \leq \ldots$ has unique factorization

$$
f(z)=e^{q(z)} P(z),
$$

for some polynomial $q(z)$ of degree not exceeding $\rho$ and infinite product $P(z)=$ $\prod_{n=1}^{\infty} E_{p}\left(\frac{z}{z_{n}}\right)$ of genus

$$
p:=\min \left\{k \in \mathbb{N}_{0}: \sum_{n=1}^{\infty} \frac{1}{\left|z_{n}\right|^{k}}=\infty \text { and } \sum_{n=1}^{\infty} \frac{1}{\left|z_{n}\right|^{k+1}}<\infty\right\} .
$$

Here $E_{p}$ denotes Weierstrass' canonical factor of order $p$, that is, $E_{p}(z)=1-z$ when $p=0$ and $E_{p}(z)=(1-z) e^{z+\frac{z^{2}}{2}+\cdots+\frac{z^{p}}{p}}$ when $p \in \mathbb{N}$, where $\mathbb{N}$ and $\mathbb{N}_{0}$ denote the sets of positive integers and of non-negative integers, respectively. In particular, each $\Phi \in H(\mathbb{C})$ of exponential type with $|\Phi(0)|=1$ and which is not zero-free has unique factorization

$$
\Phi(z)=e^{a z+b} P(z)
$$

for some $a, b \in \mathbb{C}$ with $\operatorname{Re}(b)=0$, where

$$
P(z)= \begin{cases}\prod_{n=1}^{\infty}\left(1-\frac{z}{z_{n}}\right) & \text { if } \sum_{n=1}^{\infty}\left|z_{n}\right|^{-1}<\infty, \\ \prod_{n=1}^{\infty}\left\{\left(1-\frac{z}{z_{n}}\right) e^{\frac{z}{z_{n}}}\right\} & \text { if } \sum_{n=1}^{\infty}\left|z_{n}\right|^{-1}=\infty,\end{cases}
$$

and where $\left(z_{n}\right)$ is the (possibly finite) zero-set of $\Phi$, listed with multiplicity.

\section{Proof of Theorem 1.3}

We show Theorem 1.3 by means of the following sufficient criteria for the existence of hypercyclic algebras.

Lemma 2.1. (Bayart and Matheron [4, Remark 8.26]) Let $T$ be an operator on a separable $F$-algebra $X$ so that for each triple $(U, V, W)$ of non-empty open subsets of $X$ with $0 \in W$ and for each $m \in \mathbb{N}$ there exists $f \in U$ and $q \in \mathbb{N}$ so that

$$
\left\{\begin{array}{l}
T^{q}\left(f^{j}\right) \in W \quad \text { for } 0 \leq j<m \\
T^{q}\left(f^{m}\right) \in V
\end{array}\right.
$$

Then the generic element of $X$ generates a hypercyclic algebra for $T$.

In order to apply Lemma 2.1 we first establish the next two lemmas.

Lemma 2.2. Let $A_{1}, A_{2}$ be complex scalars, where $A_{2}$ is non-zero. Then there exists $\theta \in \mathbb{R}$ so that

$$
\begin{aligned}
& 0 \leq \operatorname{Re}\left(A_{1} e^{i \theta}\right) \\
& 0<\operatorname{Re}\left(A_{2} e^{i 2 \theta}\right) .
\end{aligned}
$$

Moreover, if $A_{1}$ is also non-zero, then we may conclude both inequalities in (2.2) to be strict.

Proof. If $A_{1}=0$ the conclusion follows for example for $\theta=\frac{1}{2} \operatorname{Arg}\left(\overline{A_{2}}\right)$. If $A_{1} \neq 0$, we may select $\theta$ from the following table, according to which quadrants $A_{1}$ and $A_{2}$ belong to.

For example, if both $A_{1}$ and $A_{2}$ lie in the first quadrant we may choose $\theta=0$. If $A_{1}$ and $A_{2}$ are in the second and first quadrant respectively, then we may choose 


\begin{tabular}{|c|cccc|}
\hline$A_{2} A_{1}$ & I & II & III & IV \\
\hline I & 0 & $\pi^{+}$ & $\pi$ & 0 \\
II & $\left(-\frac{\pi}{2}\right)^{+}$ & $\left(-\frac{\pi}{2}\right)^{+}$ & $\left(\frac{\pi}{2}\right)^{+}$ & $\left(\frac{\pi}{2}\right)^{+}$ \\
III & $\left(-\frac{\pi}{2}\right)^{+}$ & $-\frac{\pi}{2}$ & $\frac{\pi}{2}$ & $\frac{\pi}{2}$ \\
IV & $0^{+}$ & $\pi^{+}$ & $\pi^{+}$ & $0^{+}$ \\
\hline
\end{tabular}

$\theta=\pi^{+}$, that is, $\theta$ close to $\pi$ and slightly larger than $\pi$. For the purpose of using this table we understand the first quadrant QI to be given by

$$
\mathrm{QI}:=\{z \in \mathbb{C}: \operatorname{Re}(z)>0 \text { and } \operatorname{Im}(z) \geq 0\},
$$

and the remaining quadrants to be given similarly:

$$
\begin{aligned}
& \text { QII }:=\{z \in \mathbb{C}: \operatorname{Re}(z) \leq 0 \text { and } \operatorname{Im}(z)>0\} \\
& \text { QIII }:=\{z \in \mathbb{C}: \operatorname{Re}(z)<0 \text { and } \operatorname{Im}(z) \leq 0\} \\
& \text { QIV }:=\{z \in \mathbb{C}: \operatorname{Re}(z) \geq 0 \text { and } \operatorname{Im}(z)<0\} .
\end{aligned}
$$

The conclusion of the lemma now follows.

Lemma 2.3. Let $\Phi$ be entire and let $w_{0}$ so that

$$
\Phi\left(w_{0}\right) \neq 0 \quad \text { and } \quad \Phi^{\prime \prime}\left(w_{0}\right) \Phi\left(w_{0}\right) \neq \Phi^{\prime}\left(w_{0}\right)^{2} .
$$

Then we have:

(a) For each $\delta>0$ there exists $w_{1}$ in $D\left(w_{0}, \delta\right)$ so that the function

$$
\left[w_{0}, w_{1}\right] \rightarrow \mathbb{R}, z \mapsto \log |\Phi(z)|,
$$

is strictly convex and $\log |\Phi(z)|$ strictly increases as $z$ moves from $w_{0}$ to $w_{1}$. That is, the function

$$
t \mapsto \log \left|\Phi\left((1-t) w_{0}+t w_{1}\right)\right|,
$$

is both strictly convex and strictly increasing on $[0,1]$.

(b) If in addition to (2.3) we also assume $\Phi^{\prime}\left(w_{0}\right) \neq 0$, then we may also conclude that

$$
t \mapsto \log \left|\Phi\left((1-t) w_{0}+t w_{1}\right)\right|
$$

is both strictly increasing and strictly convex on $[-1,1]$.

Proof. Reducing $\delta>0$ if necessary, we may assume that $\Phi$ is zero-free on $D\left(w_{0}, \delta\right)$, and thus that

$$
\Phi(z)=e^{h(z)} \quad z \in D\left(w_{0}, \delta\right)
$$

for some $h=h(z)$ holomorphic on $D\left(w_{0}, \delta\right)$. By our assumption we have

$$
h^{\prime \prime}\left(w_{0}\right)=\frac{\Phi^{\prime \prime}\left(w_{0}\right) \Phi\left(w_{0}\right)-\Phi^{\prime}\left(w_{0}\right)^{2}}{\Phi\left(w_{0}\right)^{2}} \neq 0,
$$

so we can write

$$
h(z)=a_{0}+a_{1}\left(z-w_{0}\right)+a_{2}\left(z-w_{0}\right)^{2}+\cdots \quad\left(z \in D\left(w_{0}, \delta\right)\right),
$$

with $a_{2} \neq 0$. By Lemma 2.2 we may choose $\theta \in \mathbb{R}$ so that

$$
\operatorname{Re}\left(a_{1} e^{i \theta}\right) \geq 0 \text { and } \operatorname{Re}\left(a_{2} e^{i 2 \theta}\right)>0 .
$$


Then the function

$$
g:(-\delta, \delta) \rightarrow \mathbb{R}, g(t)=\operatorname{Re}(h)\left(w_{0}+t e^{i \theta}\right)=\sum_{n=0}^{\infty} \operatorname{Re}\left(a_{n} e^{i n \theta}\right) t^{n},
$$

is smooth and $g^{\prime \prime}(0)>0$ by our selection of $\theta$. So $g^{\prime \prime}>0$ on some $[-\rho, \rho] \subset(-\delta, \delta)$ with $\rho>0$ and since $g^{\prime}(0)=\operatorname{Re}\left(a_{1} e^{i \theta}\right) \geq 0$ there must exist $\eta$ in $(0, \rho)$ so that $g$ is strictly increasing on $[0, \eta]$ as well. Hence

$$
G:[0,1] \rightarrow \mathbb{R}, G(t):=g(\eta t)
$$

is both strictly convex and strictly increasing on $[0,1]$. But for $w_{1}:=w_{0}+\eta e^{i \theta}$ we have

$$
G(t)=\log \left|\Phi\left(w_{0}+t \eta e^{i \theta}\right)\right|=\log \left|\Phi\left((1-t) w_{0}+t w_{1}\right)\right| \quad(t \in[0,1]),
$$

and $(a)$ holds. To show $(b)$ we proceed similarly, noting that the new assumption $\Phi^{\prime}\left(w_{0}\right) \neq 0$ gives that the expansion of $h$ in (2.6) also satisfies $a_{1} \neq 0$, and now the angle $\theta$ can be chosen so that both points $a_{1} e^{i \theta}$ and $a_{2} e^{i 2 \theta}$ in (2.7) have strictly positive real part, and thus the function $g$ in (2.8) has strictly positive first and second derivatives at the origin. Choosing $\rho \in(0, \delta)$ so that both $g^{\prime}$ and $g^{\prime \prime}$ are strictly positive on $[-\rho, \rho]$, the conclusion $(b)$ now follows for $w_{1}:=w_{0}+\rho e^{i \theta}$, where $\log \left|\Phi\left((1-t) w_{0}+t w_{1}\right)\right|=G(t)=g(\rho t)$ for $t \in[-1,1]$ in this case.

We are now ready to show Theorem 1.3

Proof of Theorem 1.3. Let $U, V$, and $W$ be non-empty open subsets of $H(\mathbb{C})$ with $0 \in W$, and let $m \geq 2$ be given. By Lemma2.1 it suffices to find some $(f, q) \in U \times \mathbb{N}$ so that

$$
\Phi(D)^{q}\left(f^{m}\right) \in V \quad \text { and } \quad \Phi(D)^{q}\left(f^{j}\right) \in W(1 \leq j<m) .
$$

Get $w$ a non-zero scalar so that $\{w, 2 w, \ldots m w\} \subset \Phi^{-1}(\mathbb{D})$, and chose $\delta>0$ small enough 11 so that for each $s \in\{1, \ldots, m\}$ and $w_{1}, \ldots, w_{s} \in D(w, \delta)$ and for each $d \in\{0, \ldots, m-s\}$ and $v_{1}, \ldots, v_{d} \in D(0, \delta)$ we have

$$
w_{1}+\cdots+w_{s}+v_{1}+\cdots+v_{d} \in \Phi^{-1}(\mathbb{D}),
$$

where the expression $v_{1}+\cdots+v_{d}$ is defined as zero whenever $d=0$. Now, by Lemma 2.3 there exists a non-zero scalar $w^{*}$ in $D(0, \delta)$ so that the map

$$
\left[0, w^{*}\right] \rightarrow \mathbb{R}, \quad z \mapsto \log |\Phi(z)|,
$$

is strictly convex, and it increases with $|z|$ whenever $z \in\left[0, w_{0}\right]$, for some $w_{0} \in$ $\left(0, w^{*}\right)$. Now, since the sets $D(w, \delta)$ and $\left[\frac{w_{0}}{2}, w_{0}\right]$ have accumulation points in $\mathbb{C}$

\footnotetext{
${ }^{1}$ To see why such $\delta>0$ exists: Let $g: \mathbb{C}^{m} \rightarrow \mathbb{C}, g\left(z_{1}, \ldots, z_{m}\right):=\Phi\left(z_{1}+\cdots+z_{m}\right)$. By our assumption, for each $s \in\{1, \ldots, m\}$ we have $g(\underbrace{w, \ldots, w}, 0, \ldots, 0)=\Phi(s w) \in \mathbb{D}$. The continuity of $g$ ensures the existence of some $\delta_{s}>0$ so that

$$
\underbrace{D\left(w, \delta_{s}\right) \times \cdots \times D\left(w, \delta_{s}\right)}_{s} \times \underbrace{D\left(0, \delta_{s}\right) \times \cdots \times D\left(0, \delta_{s}\right)}_{m-s} \subset \Phi^{-1}(\mathbb{D}) .
$$

In particular, for each $w_{1}, \ldots, w_{s} \in D\left(w, \delta_{s}\right)$ and $v_{1}, \ldots, v_{d} \in D\left(0, \delta_{s}\right)$ with $s+d \leq m$ we have

$$
\Phi\left(w_{1}+\cdots+w_{s}+v_{1}+\cdots+v_{d}\right)=g\left(w_{1}, \ldots, w_{s}, v_{1}, \ldots, v_{d}, 0, \ldots, 0\right) \in \mathbb{D} .
$$
}

Then $\delta:=\min \left\{d_{s}: 1 \leq s \leq m\right\}$ does the job. 
there exist $p \in \mathbb{N}, \alpha_{1}, \ldots, \alpha_{p} \in D(w, \delta)$, and $\beta_{1}, \ldots, \beta_{p} \in\left[\frac{w_{0}}{2}, w_{0}\right]$ and scalars $a_{i}, b_{i} \in \mathbb{C} \backslash\{0\}, i \in I_{p}:=\{1, \ldots, p\}$, so that

$$
(A, B):=\left(\sum_{i \in I_{p}} a_{i} e^{\alpha_{i} z}, \sum_{i \in I_{p}} b_{i} e^{\beta_{i} z}\right) \in U \times V .
$$

Next, set $R_{N}:=\sum_{i=1}^{p} c_{i} e^{\lambda_{i} z}$, where $\left(m \lambda_{1}, \ldots, m \lambda_{p}\right)=\left(\beta_{1}, \ldots, \beta_{p}\right)$ and where $\left(c_{1}, \ldots, c_{p}\right)=\left(c_{1}(N), \ldots, c_{p}(N)\right)$ is a solution of the system

$$
b_{i}=c_{i}^{m} \Phi\left(m \lambda_{i}\right)^{N} \quad\left(i \in I_{p}\right) .
$$

Notice that for each $i \in I_{p}$ we have $c_{i}=c_{i}(N) \underset{N \rightarrow \infty}{\rightarrow} 0$, thanks to having $\left|\Phi\left(m \lambda_{i}\right)\right|=$ $\left|\Phi\left(\beta_{i}\right)\right|>1$ and $b_{i} \neq 0$. So $f=f_{N}:=\left(A+R_{N}\right)$ belongs to $U$ whenever $N$ is large enough.

Now, let $\mathbb{E}_{p}:=\left\{e_{1}, \ldots, e_{p}\right\}$ denote the canonical basis of $\mathbb{C}^{p}$, and for any $x=$ $\left(x_{1}, \ldots, x_{p}\right) \in \mathbb{N}_{0}^{p}$ let $|x|:=\sum_{i \in I_{p}} x_{i}$. Also, given $x, y \in \mathbb{N}_{0}^{p}$ we let $x y:=\sum_{i \in I_{p}} x_{i} y_{i}$. Finally, for each $n \in \mathbb{N}$ we let $\mathcal{L}_{n}$ denote the set of tuples $(u, v)$ in $\mathbb{N}_{0}^{p} \times \mathbb{N}_{0}^{p}$ for which $|u|+|v|=n$. Our selection of $c_{i}$ 's and $\lambda_{i}$ 's ensures that

$$
\left\{\begin{array}{l}
\Phi(D)^{N}\left(f^{m}\right)=B+\sum_{(u, v) \in \mathcal{L}_{m}^{*}} S(u, v, N) e^{(\alpha u+\lambda v) z} \\
\Phi(D)^{N}\left(f^{n}\right)=\sum_{(u, v) \in \mathcal{L}_{n}} S(u, v, N) e^{(\alpha u+\lambda v) z} \quad(1 \leq n<m)
\end{array}\right.
$$

where

$$
\mathcal{L}_{m}^{*}:=\mathcal{L}_{m} \backslash\left\{(u, v) \in \mathcal{L}_{m}:|u|=0 \text { and } v \in m E_{p}\right\}
$$

and where for each fixed $(u, v) \in \mathcal{L}:=\mathcal{L}_{m}^{*} \cup \mathcal{L}_{1} \cup \cdots \cup \mathcal{L}_{m-1}$ there exists a scalar $\gamma=\gamma_{u, v}$ that does not depend 2 on $N$ so that

$$
S(u, v, N)=\gamma \Phi(\alpha u+\lambda v)^{N} \prod_{i \in I_{p}} c_{i}^{v_{i}}
$$

Pending is to show that for each $(u, v) \in \mathcal{L}$ we have

$$
S(u, v, N) \underset{N \rightarrow \infty}{\rightarrow} 0 .
$$

Now, let $(u, v) \in \mathcal{L}$ be fixed. By (2.11) and (2.12) we have

$$
|S(u, v, N)|=\mathcal{O}\left(\theta_{u, v}^{N}\right) \quad \text { as } N \rightarrow \infty,
$$

where $\theta_{u, v}=\frac{|\Phi(\alpha u+\lambda v)|}{\prod_{i \in I_{p}}\left|\Phi\left(m \lambda_{i}\right)\right|^{v_{i} / m}}$. So it suffices to verify that $\theta_{u, v} \in[0,1)$. We have three cases:

Case 1: $\quad(u, v) \in \mathcal{L}_{m}^{*}$ with $|u|=0$. Here $|v|=m$ and $v \notin m \mathbb{E}_{p}$, so $\lambda v$ is a non-trivial convex combination of $m \lambda_{1}, \ldots, m \lambda_{p}$. Hence

$$
\theta_{u, v}=\frac{|\Phi(\lambda v)|}{\prod_{i \in I_{p}}\left|\Phi\left(m \lambda_{i}\right)\right|^{v_{i} / m}}<1
$$

thanks to the map $z \mapsto \log |\Phi(z)|$ being strictly convex on $\left[0, w^{*}\right]$ and our selection of the $\lambda_{i}$ 's.

Case 2: $1 \leq|u| \leq m$ and $|v| \leq m-|u|$. By (2.10) we have $\alpha u+\lambda v$ in $\Phi^{-1}(\mathbb{D})$. Also, since $|\Phi(0)|=1$ and since $z \mapsto \log |\Phi(z)|$ strictly increases with $|z|$ whenever $z \in\left[0, w_{0}\right]$, we have that $\left|\Phi\left(m \lambda_{i}\right)\right|>1$ for each $i \in I_{p}$. So $\theta_{u, v} \in[0,1)$.

\footnotetext{
${ }^{2}$ Indeed, the multinomial theorem gives $\gamma_{u, v}=(|u|+|v|) ! \prod_{i=1}^{p} \frac{a_{i}^{u_{i}}}{u_{i} ! v_{i} !}$
} 
Case 3: $|u|=0$ and $1 \leq|v| \leq m-1$. Notice that in this case

$$
\alpha u+\lambda v=0\left(1-\frac{|v|}{m}\right)+m \lambda_{1} \frac{v_{1}}{m}+\cdots+m \lambda_{p} \frac{v_{p}}{m}
$$

is a non-trivial convex combination of the points $0, m \lambda_{1}, \ldots, m \lambda_{p}$ lying on $\left[0, w^{*}\right]$. So the strict convexity of $z \mapsto \log |\Phi(z)|$ on $\left[0, w^{*}\right]$ and the fact that $|\Phi(0)|=1$ give

$$
\begin{aligned}
|\Phi(\alpha u+\lambda v)| & <|\Phi(0)|^{1-\frac{|v|}{m}} \prod_{i \in I_{p}}\left|\Phi\left(m \lambda_{i}\right)\right|^{v_{i} / m} \\
& =\prod_{i \in I_{p}}\left|\Phi\left(m \lambda_{i}\right)\right|^{v_{i} / m}
\end{aligned}
$$

and thus that $\theta_{u, v} \in[0,1)$.

The proof of Theorem 1.3. is now complete.

\section{Proofs of Theorem 1.6 and Theorem 1.2 ,}

We use the following result by Bayart to show Theorem 1.6 .

Theorem 3.1. (Bayart) [3, pp 3452-3454] Let $\Phi$ be entire and of exponential type. Suppose that for each $m \geq 2$ there exist scalars $z_{0}$ and $w_{0}$ with $z_{0} \in\left(0, w_{0}\right)$ so that

(a) $\left|\Phi\left(w_{0}\right)\right|>1$ and $|\Phi|<1$ on $\left(0, z_{0}\right]$,

(b) $\left|\Phi\left(d w_{0}\right)\right|<\left|\Phi\left(w_{0}\right)\right|^{d}$ for each $d \in\{2,3, \ldots, m\}$, and

(c) The function $t \mapsto\left|\Phi\left(w_{0}+t z_{0}\right)\right|$ is strictly increasing on $[0, \eta)$, for some $\eta>0$.

Then $\Phi(D)$ supports a hypercyclic algebra on $H(\mathbb{C})$.

We also use the following elementary fact:

Remark 3.2. Given $f:[a, b] \rightarrow \mathbb{R}$ continuously differentiable and $y \in(f(a), f(b))$, there exists $c \in(a, b)$ so that $y<f$ on $[c, b]$ and $f^{\prime}(c)>0$.

Proof of Theorem 1.6. Fix $m \geq 2$. By (i) and Theorem 3.1, it suffices to show that there exists some $w_{0} \in\left\{t z_{0}: t>1\right\}$ so that

(a') $\left|\Phi\left(w_{0}\right)\right|>1$,

(b') $\left|\Phi\left(d w_{0}\right)\right|<\left|\Phi\left(w_{0}\right)\right|^{d}$ for each $d \in\{2,3, \ldots, m\}$, and

(c') The function $t \mapsto\left|\Phi\left(w_{0}+t z_{0}\right)\right|$ is strictly increasing on an interva $3(-\eta, \eta)$, for some $\eta>0$.

By means of contradiction, suppose no such $w_{0}$ satisfies $\left(a^{\prime}\right)-\left(c^{\prime}\right)$. Let $t^{*}>1$ so that $z_{1}=t^{*} z_{0}$. Also, consider the smooth function $\psi: \mathbb{R} \rightarrow \mathbb{R}, \psi(t)=\left|\Phi\left(t z_{0}\right)\right|^{2}$. By (ii) we may pick $0<\epsilon<\psi\left(t^{*}\right)-\max \left\{1, e^{2 \tau_{0}\left|z_{1}\right|}\right\}$. Also, let $\frac{1}{4}>\epsilon_{1}>\epsilon_{2}>\cdots>0$ so that

$$
\prod_{k=1}^{\infty}\left(1-\epsilon_{k}\right)>1-\epsilon
$$

By $(i i)$ we have

$$
\psi(1)<1 \leq \max \left\{1, e^{2 \tau_{0}\left|z_{1}\right|}\right\}<\psi\left(t^{*}\right) .
$$

\footnotetext{
${ }^{3}$ While we only need $[0, \eta)$ in (c') for this proof, we later use in the proof of Theorem 4.4 this stronger conclusion of having $(-\eta, \eta)$ in $\left(c^{\prime}\right)$.
} 
So by Remark 3.2 there exists $t_{0} \in\left(1, t^{*}\right)$ so that

$$
\begin{aligned}
\max \left\{1, e^{2 \tau_{0}\left|z_{1}\right|}\right\}<\psi\left(t^{*}\right)- & \epsilon<\left(t_{0}\right) . \\
0 & <\psi^{\prime}\left(t_{0}\right) .
\end{aligned}
$$

In particular, $1<\psi\left(t_{0}\right)=\left|\Phi\left(t_{0} z_{0}\right)\right|^{2}$ and $\psi$ is strictly increasing on $\left(t_{0}-\eta, t_{0}+\eta\right)$ for some $\eta>0$; in other words the function $t \mapsto\left|\Phi\left(\left(t+t_{0}\right) z_{0}\right)\right|=\left|\Phi\left(t_{0} z_{0}+t z_{0}\right)\right|$ is strictly increasing on $(-\eta, \eta)$. That is, $w_{0}:=t_{0} z_{0}$ satisfies $(a)$ and $(c)$ and hence it must fail (b) so for some $d_{1} \in\{2, \ldots, m\}$ we have

$$
\psi\left(d_{1} t_{0}\right) \geq \psi\left(t_{0}\right)^{d_{1}}>\psi\left(t_{0}\right)^{d_{1}\left(1-\epsilon_{1}\right)}>\psi\left(t_{0}\right) .
$$

By Remark 3.2, there exists $t_{1} \in\left(t_{0}, d_{1} t_{0}\right)$ so that

$$
\begin{aligned}
\psi\left(t_{0}\right)^{d_{1}\left(1-\epsilon_{1}\right)} & <\psi\left(t_{1}\right) \\
0 & <\psi^{\prime}\left(t_{1}\right) .
\end{aligned}
$$

Let $r_{1} \in\left(1, d_{1}\right)$ so that $t_{1}=r_{1} t_{0}$. As before $w_{0}:=t_{1} z_{0}$ satisfies $(a)$ and $(c)$, so there exists $d_{2} \in\{2, \ldots, m\}$ so that

$$
\psi\left(d_{2} t_{1}\right) \geq \psi\left(t_{1}\right)^{d_{2}}>\psi\left(t_{1}\right)^{d_{2}\left(1-\epsilon_{2}\right)}>\psi\left(t_{0}\right)^{d_{1}\left(1-\epsilon_{1}\right) d_{2}\left(1-\epsilon_{2}\right)}>\psi\left(t_{0}\right) .
$$

By Remark 3.2 there exists $t_{2} \in\left(t_{1}, d_{2} t_{1}\right)$ so that

$$
\begin{aligned}
\psi\left(t_{1}\right)^{d_{2}\left(1-\epsilon_{2}\right)} & <\psi\left(t_{2}\right) \\
0 & <\psi^{\prime}\left(t_{2}\right) .
\end{aligned}
$$

We let $r_{2} \in\left(1, d_{2}\right)$ so that $t_{2}=r_{2} t_{1}$, and note again that $w_{0}:=t_{2} z_{0}$ satisfies $(a)$ and $(c)$ to get $d_{3} \in\{2, \ldots, m\}$ with

$$
\psi\left(d_{3} t_{2}\right) \geq \psi\left(t_{2}\right)^{d_{3}}>\psi\left(t_{2}\right)^{d_{3}\left(1-\epsilon_{3}\right)}>\psi\left(t_{2}\right) .
$$

Continuing this process inductively, we obtain sequences $\left(t_{n}\right)_{n=1}^{\infty},\left(d_{n}\right)_{n=1}^{\infty}$, and $\left(r_{n}\right)_{n=1}^{\infty}$ so that for each $n \geq 1$ we have

$$
\begin{aligned}
d_{n} & \in\{2, \ldots, m\} \\
r_{n} & \in\left(1, d_{n}\right) \\
t_{n} & =r_{n} t_{n-1} \\
\psi\left(t_{n}\right) & >\psi\left(t_{n-1}\right)^{d_{n}\left(1-\epsilon_{n}\right)}>\psi\left(t_{0}\right)^{(3 / 2)^{n}} \\
\psi^{\prime}\left(t_{n}\right) & >0 .
\end{aligned}
$$

In particular, $\psi\left(t_{n}\right) \rightarrow \infty$ since $\psi\left(t_{0}\right)>1$, and thus $t_{n} \rightarrow \infty$. Also, by (3.4) we have $t_{n}=r_{n} t_{n-1}=\cdots=\left(r_{n} r_{n-1} \cdots r_{1}\right) t_{0}$ and

$$
d_{1} \cdots d_{n} \prod_{k=1}^{n}\left(1-\epsilon_{k}\right)>d_{1} \cdots d_{n}(1-\epsilon)>r_{1} \cdots r_{n}(1-\epsilon)=(1-\epsilon) \frac{t_{n}}{t_{0}},
$$

giving

$$
\begin{aligned}
\psi\left(t_{n}\right) & >\psi\left(t_{n-1}\right)^{d_{n}\left(1-\epsilon_{n}\right)} \\
& >\psi\left(t_{n-2}\right)^{d_{n}\left(1-\epsilon_{n}\right) d_{n-1}\left(1-\epsilon_{n-1}\right)} \\
& \vdots \\
& >\psi\left(t_{0}\right)^{d_{1} \cdots d_{n} \prod_{k=1}^{n}\left(1-\epsilon_{k}\right)} \\
& >\psi\left(t_{0}\right)^{(1-\epsilon) \frac{t_{n}}{t_{0}}}
\end{aligned}
$$


So for each $n \geq 1$

$$
\begin{aligned}
\left|\Phi\left(t_{n} z_{0}\right)\right| & >\left|\Phi\left(t_{0} z_{0}\right)\right|^{(1-\epsilon) \frac{t_{n}}{t_{0}}} \\
& =e^{(1-\epsilon) \log \left|\Phi\left(t_{0} z_{0}\right)\right| \frac{t_{n}}{t_{0}}} \\
& >e^{\left.(1-\epsilon) \log \left(\left(\left|\Phi\left(t^{*} z_{0}\right)\right|^{2}-\epsilon\right)^{\frac{1}{2}}\right) \frac{t_{n}}{t_{0}} \quad \text { by [3.1) }\right)} \\
& >e^{(1-\epsilon) \frac{\log \left(\left(\left|\Phi\left(z_{1}\right)\right|^{2}-\epsilon\right) \frac{1}{2}\right.}{\left|z_{1}\right|} t_{n}\left|z_{0}\right|} \\
& =e^{(1-\epsilon) \frac{\log \left(\left(\left|\Phi\left(t^{*} z_{0}\right)\right|^{2}-\epsilon\right) \frac{1}{2}\right)}{t^{*} z_{0} \mid} t_{n}\left|z_{0}\right|}
\end{aligned}
$$

Hence for each small $\epsilon>0$ we have

$$
\tau_{0} \geq(1-\epsilon) \frac{\log \left(\left(\left|\Phi\left(z_{1}\right)\right|^{2}-\epsilon\right)^{\frac{1}{2}}\right)}{\left|z_{1}\right|}
$$

forcing $\tau_{0} \geq \frac{\log \left|\Phi\left(z_{1}\right)\right|}{\left|z_{1}\right|}$, contradicting our assumption (1.1).

Corollary 3.3. Let $\Phi(z)=e^{a z} \varphi(z)$, where $\varphi$ is non-constant of growth $\left(\frac{1}{2}, 0\right)$ with $|\varphi(0)|=1$, and $\operatorname{Im}\left(a \varphi(0) \overline{\varphi^{\prime}(0)}\right) \neq 0$. Then $\Phi(D)$ supports a hypercyclic algebra.

To show this we recall the following fact.

Lemma 3.4. [9, Lemma 5] Let $\Phi \in H(\mathbb{C})$ be of exponential type, and consider the composition operator $C_{\varphi}: H(\mathbb{C}) \rightarrow H(\mathbb{C}), f \mapsto f \circ \varphi$, where $\varphi: \mathbb{C} \rightarrow \mathbb{C}, \varphi(z)=a z$, is a homothety on the plane with $0 \neq a \in \mathbb{C}$. Then $\Phi_{a}:=C_{\varphi}(\Phi)$ is of exponential type and

$$
C_{\varphi}\left(H C\left(\Phi_{a}(D)\right)\right)=H C(\Phi(D)) .
$$

In particular, the algebra isomorphism $C_{\varphi}: H(\mathbb{C}) \rightarrow H(\mathbb{C})$ maps hypercyclic algebras of $\Phi_{a}(D)$ onto hypercyclic algebras of $\Phi(D)$.

Proof of Corollary 3.3. By Lemma 3.4 the convolution operator $\Phi(D)$ has the same set of hypercyclic vectors as the one induced by

$$
\Phi_{a^{-1}}(z)=\Phi\left(\frac{z}{a}\right)=e^{z} \varphi_{a^{-1}}(z),
$$

where $\varphi_{a^{-1}}(z)=\varphi\left(\frac{z}{a}\right)$. Notice that $\varphi_{a^{-1}}$ is also of subexponential growth and that it is of growth $\left(\frac{1}{2}, 0\right)$ whenever $\varphi$ is, and that $\left|\Phi_{a^{-1}}\right|=\left|\varphi_{a^{-1}}\right|$ on the imaginary axis. It suffices to show $\Phi_{a^{-1}}$ satisfies the assumptions of Theorem 1.6. Letting $g(t):=\left|\Phi_{a^{-1}}(i t)\right|^{2}=\left|\varphi\left(\frac{i t}{a}\right)\right|^{2}$, we have $g^{\prime}(0)=\frac{2}{|a|^{2}} \operatorname{Im}\left(a \varphi(0) \overline{\varphi^{\prime}(0)}\right) \neq 0$ and thus there exists some $\eta>0$ so that $\Phi_{a^{-1}}$ has modulus smaller than one on $(0, \eta i)$ if $g^{\prime}(0)<0$ or on $(-\eta i, 0)$ if $g^{\prime}(0)>0$. Also $\varphi_{a^{-1}}$ is unbounded on any half line, since it is of growth $\left(\frac{1}{2}, 0\right)$ [11, Theorem 3.1.5].

Corollary 3.5. Let $\Phi(z)=e^{a z} \prod_{n=1}^{\infty}\left(1-\frac{z}{z_{n}}\right)$, where $a$ and $z_{n}(n \in \mathbb{N})$ are non-zero complex scalars satisfying

(i) $\sum_{n=1}^{\infty} \frac{1}{\left|z_{n}\right|}<\infty$

(ii) $\sum_{n=1}^{\infty} \frac{1}{z_{n}^{2}} \neq 0$.

Then $\Phi(D)$ has a hypercyclic algebra. 
Proof. Condition $(i)$ ensures that $P(z):=\prod_{n=1}^{\infty}\left(1-\frac{z}{z_{n}}\right)$ converges absolutely and locally uniformly on $\mathbb{C}$, and that $P$ is of growth $(1,0)$ [11, Lemma 2.10.13]. Hence $\Phi$ is non-constant of exponential type. By Lemma 3.4. replacing $\left(z_{n}\right)$ by $\left(a z_{n}\right)$ if necessary, without loss of generality we may assume that $a=1$. We show that $\Phi(z)=e^{z} P(z)$ satisfies the assumptions of Theorem 1.3. Notice first that $\Phi^{\prime \prime}(0) \Phi(0) \neq \Phi^{\prime}(0)^{2}$ if and only

$$
P^{\prime \prime}(0) P(0) \neq P^{\prime}(0)^{2},
$$

and that $P(0)=1, P^{\prime}(0)=-\sum_{n=1}^{\infty} \frac{1}{z_{n}}$, and $P^{\prime \prime}(0)=\left(-\sum_{n=1}^{\infty} \frac{1}{z_{n}}\right)^{2}+\sum_{n=1}^{\infty} \frac{1}{z_{n}^{2}}$. So (3.5) holds precisely when (ii) does. Finally, since $P$ is of growth $(1,0)$ we have $\lim _{x \rightarrow-\infty} e^{x} P(x)=0$, so $\Phi^{-1}(\mathbb{D})$ contains arithmetic progressions of the desired form.

The case $a=0$ of Corollary 3.5 is covered by Corollary 3.6 .

Corollary 3.6. Let $\Phi \in H(\mathbb{C})$ be non-constant and of subexponential growth, with $|\Phi(0)|=1$. Then $\Phi(D)$ has a hypercyclic algebra.

Proof. By Theorem 1.6 it suffices to show that there exist $z_{1} \in \mathbb{C}$ and $0<\delta<1$ so that

$$
\left\{\begin{array}{c}
|\Phi|<1 \text { on }\left(0, \delta z_{1}\right] \\
\left|\Phi\left(z_{1}\right)\right|>\max \left\{1, e^{\tau_{0}\left|z_{1}\right|}\right\}
\end{array}\right.
$$

where $\tau_{0}=\tau_{0}\left(z_{1}\right)=\lim \sup _{r \rightarrow \infty} r^{-1} \log \left|\Phi\left(r \frac{z_{1}}{\left|z_{1}\right|}\right)\right|$. Since $\Phi \in H(\mathbb{C})$ is nonconstant, we may write

$$
\Phi(z)=\Phi(0)+a_{N} z^{N}+o\left(Z^{N}\right) \quad(z \rightarrow 0),
$$

with $N \geq 1$ and $a_{N} \neq 0$. Notice also that for each $A \in \mathbb{C} \backslash\{0\}$ and each $\theta \in \mathbb{R}$ we have that $\Phi$ satisfies (3.6) for $\left(z_{1}, \delta, \tau\right)$ if and only if

$$
\widetilde{\Phi}(z)=e^{i \theta} \Phi(A z)
$$

satisfies (3.6) for $\left(\widetilde{z}_{1}, \widetilde{\delta}, \widetilde{\tau}\right)=\left(\frac{z_{1}}{A}, \delta,|A| \tau\right)$. So taking $\theta \in \mathbb{R}$ and $A \in \mathbb{C}$ of modulus one so that

$$
\begin{aligned}
e^{i \theta} \Phi(0) & =1 \\
e^{i \theta} a_{N} A^{N} & <0,
\end{aligned}
$$

without loss of generality we may assume that

$$
\Phi(z)=1-a z^{N}+o\left(z^{N}\right) \quad(z \rightarrow 0)
$$

where $a>0$ and $N \geq 1$. Since $\Phi$ is of growth $(1,0)$ it can't be bounded on any open half-plane. So there exists some $z_{0} \in \mathbb{C}$ with

$$
\operatorname{Re}\left(z_{0}\right)>0 \text { and }\left|\Phi\left(z_{0}\right)\right|>1
$$

Now, for each $0 \leq j<N$ let $\Phi_{j}(z):=\Phi\left(w^{j} z\right)$, where $w:=e^{i \frac{2 \pi}{N}}$. Notice that each $\Phi_{j}$ is of growth $(1,0)$ and satisfies (3.7). Hence replacing $\Phi$ by $\Phi_{j}$ for some $0 \leq j<N$ if necessary, without loss of generality we may assume that $\operatorname{Arg}\left(z_{0}\right) \in\left(-\frac{\pi}{2 N}, \frac{\pi}{2 N}\right)$. Hence for $t \in \mathbb{R}$ we have

$$
\begin{aligned}
\Phi\left(t z_{0}\right) & =1-a t^{N} z_{0}^{N}+g(t) \\
& =1-t^{N}\left(a z_{0}^{N}-\frac{g(t)}{t^{N}}\right),
\end{aligned}
$$


where $g(t)=o\left(t^{N}\right)$ as $t \rightarrow 0$. Now, let $K$ be a closed disc centered at $a z_{0}^{N}$ and contained in $\{z \in \mathbb{C}: \operatorname{Re}(z)>0\}$. Then there exists $\delta>0$ so that for any $0<s<\delta$ the set $s K$ is contained in the open disc $D(1,1)$. Hence by (3.8) for each $0<t<\delta$ we have $\left|\Phi\left(t z_{0}\right)\right|<1$. So $z_{1}:=z_{0}$ works, because by Remark 1.7 (b) we have $\tau_{0}=0$, as $\Phi$ is of subexponential growth.

Lemma 3.7. Let $\Phi(z)=p(z) e^{z}$, where $p(z)=1+a_{1} z+a_{2} z^{2}+\cdots+a_{r} z^{r}$, with $r \geq 1$ and $a_{r} \neq 0$. Suppose that $\operatorname{Im}\left(a_{1}\right) \neq 0$ or that $2 a_{2} \neq a_{1}^{2}$. Then $\Phi(D)$ supports a hypercyclic algebra.

Proof. The case $\operatorname{Im}\left(a_{1}\right) \neq 0$ is immediate from Corollary 3.3. So assume that $a_{1} \in$ $\mathbb{R}$. We show that $\Phi$ satisfies the assumptions of Theorem 1.3. Since $|\Phi(x)| \rightarrow 0$ as $x \rightarrow-\infty$, the set $\Phi^{-1}(\mathbb{D})$ contains an interval of the form $(-\infty, x]$, and in particular the desired arithmetic progressions. But $\Phi(0)=1$ and $\Phi^{\prime \prime}(0) \Phi(0)-\Phi^{\prime}(0)^{2}=$ $2 a_{2}-a_{1}^{2} \neq 0$.

Proof of Theorem 1.2. Conclusion (a) has been established in Corollary 3.6. So assume $\Phi$ is not of subexponential growth, that is, it has growth order one and positive finite type. If $\Phi$ is zero-free it is a scalar multiple of an exponential and Conclusion (b1) holds, see 2. Conclusion (b2) follows from Lemma 3.4 and Lemma 3.7, noting that the scalar $a$ must be non-zero in this case as $\Phi$ is of order one. To show (b3), consider the Hadamard factorization

$$
\Phi(z)=e^{a z} P(z),
$$

of $\Phi$, where $P(z)=\prod_{n=1}^{\infty} E_{p}\left(\frac{z}{z_{n}}\right)$ is the canonical product of genus $p$ of the zero-set $\left(z_{n}\right)$ of $\Phi$. Notice that $p$ must be zero or one, since $\Phi$ has order one. Case (i), when $\sum_{n=1}^{\infty}\left|z_{n}\right|^{-1}<\infty$, has been established in Corollary 3.5 as $p=0$ in this case. Case (ii), where $\sum_{n=1}^{\infty}\left|z_{n}\right|^{-1}=\infty$, implies we have $p=1$ and hence

$$
P(z)=\prod_{n=1}^{\infty}\left\{\left(1-\frac{z}{z_{n}}\right) e^{\frac{z}{z_{n}}}\right\}
$$

so $P(0)=1, P^{\prime}(0)=0$, and $P^{\prime \prime}(0)=\sum_{n=1}^{\infty} z_{n}^{-2}$. So by our assumption we have $P^{\prime \prime}(0) P(0) \neq P^{\prime}(0)^{2}$, and equivalently that $\Phi^{\prime \prime}(0) \Phi(0) \neq \Phi^{\prime}(0)^{2}$. Also, $\Phi^{\prime}(0)=$ $P^{\prime}(0)+a P(0)=a \neq 0$ by our assumption on $a$, and the conclusion follows from Corollary 4.3 .

Corollary 3.8. Let $\left(z_{n}\right)$ be a sequence of complex scalars with $0<\left|z_{1}\right| \leq\left|z_{2}\right| \leq \ldots$ and satisfying

(i) $\sum_{n=1}^{\infty}\left|z_{n}\right|^{-1}$ diverges, and $\sum_{n=1}^{\infty}\left|z_{n}\right|^{-p}$ converges for each $p>1$.

(ii) $\limsup _{r \rightarrow \infty} r^{-1} \max \left\{j \in \mathbb{N}:\left|z_{j}\right| \leq r\right\}<\infty$,

(iii) The set $\left\{\sum_{\left|z_{n}\right| \leq r} z_{n}^{-1}: r>0\right\}$ is bounded, and

(iv) $\sum_{n=1}^{\infty} z_{n}^{-2} \neq 0$.

Then for each

$$
\Phi(z)=e^{a z} \prod_{n=1}^{\infty}\left\{\left(1-\frac{z}{z_{n}}\right) e^{\frac{z}{z_{n}}}\right\}
$$

with $a \neq 0$, the operator $\Phi(D)$ supports a hypercyclic algebra.

Proof. By $(i)$ the convergence exponent of the zeros of $f$ is $\rho_{1}=1$, and the canonical product $\prod_{n=1}^{\infty}\left\{\left(1-\frac{z}{z_{n}}\right) e^{\frac{z}{z_{n}}}\right\}$ has order equal to the convergence exponent of its zeros [11, Theorem 2.6.5], which is equal to 1 , by $(i)$. So $\Phi$ is of order at most 1 , and 
by $($ ii $)$ and $($ iii $)$ we may conclude $\Phi$ is of exponential type [11, Theorem 2.9.5 and Theorem 2.10.1]. The conclusion follows from Theorem 1.2.

The following consequence of Theorem 1.2 complements [9, Corollary 14].

Corollary 3.9. Let $p=p(z)$ be a non-constant polynomial with $|p(0)| \leq 1$. Then the differentiation operator $p\left(\frac{d}{d x}\right)$ acting on $\mathbb{C}^{\infty}(\mathbb{R}, \mathbb{C})$ supports a hypercyclic algebra.

Proof. The operator $p\left(\frac{d}{d x}\right)$ is quasi-conjugate to $p(D): H(\mathbb{C}) \rightarrow H(\mathbb{C})$ via a multiplicative operator, so it suffices to verify that $p(D)$ supports a hypercyclic algebra. The conclusion now follows from Theorem 1.1 when $|p(0)|<1$ and from the LeónMüller Theorem and Case (a) of Theorem 1.2 when $|p(0)|=1$.

\section{Proof of Theorem 1.8 .}

We note that the same arguments used in the proofs of Theorem 1.2 , Theorem 1.3 and Theorem 1.6 give general eigenvalue criteria for the existence of hypercyclic algebras. This general formulation was used by Bayart [3] to provide hypercyclic algebras for operators on the space $\ell_{1}(\mathbb{N})$ endowed with the convolution product, for instance. A criterion corresponding to Theorem 1.3, for example, may be stated as follows.

Theorem 4.1. Let $T$ be an operator on a separable infinite dimensional $F$-algebra $X$. Suppose there exist a function $E: \mathbb{C} \rightarrow X$ and an entire function $\phi: \mathbb{C} \rightarrow \mathbb{C}$ satisfying the following:

(a) For each $\lambda \in \mathbb{C}, T E(\lambda)=\phi(\lambda) E(\lambda)$,

(b) For each $\lambda, \mu \in \mathbb{C}, E(\lambda) E(\mu)=E(\lambda+\mu)$,

(c) For each subset $\Lambda$ of $\mathbb{C}$ supporting an accumulation point in $\mathbb{C}$, the set $\{E(\lambda): \lambda \in \Lambda\}$ has dense linear span in $X$,

(d) The function $\phi$ satisfies $|\phi(0)|=1$, and $\phi^{\prime \prime}(0) \phi(0) \neq \phi^{\prime}(0)^{2}$, and

(e) For each positive integer $m$ there exists a non-zero scalar $a=a(m)$ so that $\{a, 2 a, \ldots, m a\} \subset \phi^{-1}(\mathbb{D})$.

Then the singly generated algebra induced by a generic element $f$ of $X$ is a hypercyclic algebra for $T$.

We adopt this point of view when providing with Theorem 4.2 and Theorem 4.4 below general eigenvalue criteria for the existence of dense hypercyclic algebras induced by infinitely many free generators, and use such criteria to establish Theorem 1.8

Theorem 4.2. Let $X$ be a separable commutative $F$-algebra that supports a dense freely generated subalgebra, and let $T$ be an operator on $X$. Suppose there exist a function $E: \mathbb{C} \rightarrow X$ and an entire function $\phi: \mathbb{C} \rightarrow \mathbb{C}$ satisfying the following:

(a) For each $\lambda \in \mathbb{C}, T E(\lambda)=\phi(\lambda) E(\lambda)$,

(b) For each $\lambda, \mu \in \mathbb{C}, E(\lambda) E(\mu)=E(\lambda+\mu)$,

(c) For each subset $\Lambda$ of $\mathbb{C}$ supporting an accumulation point in $\mathbb{C}$, the set $\{E(\lambda): \lambda \in \Lambda\}$ has dense linear span in $X$, and

(d) The function $\phi$ satisfies $|\phi(0)|=1, \phi^{\prime \prime}(0) \phi(0) \neq \phi^{\prime}(0)^{2}$, and $\phi^{\prime}(0) \neq 0$.

Then the generic element $f=\left(f_{n}\right)_{n=1}^{\infty}$ of $X^{\mathbb{N}}$ freely generates a dense subalgebra of $X$ consisiting entirely (but zero) of hypercyclic vectors for $T$. 
Taking $X=H(\mathbb{C}), \Phi=\phi, T=\Phi(D)$, and $E(\lambda)(z):=e^{\lambda z}(\lambda, z \in \mathbb{C})$ in Theorem 4.2 we have the following corollary.

Corollary 4.3. Let $\Phi$ be of exponential type satisfying $|\Phi(0)|=1, \Phi^{\prime \prime}(0) \Phi(0) \neq$ $\Phi^{\prime}(0)^{2}$, and $\Phi^{\prime}(0) \neq 0$. Then the set of hypercyclic vectors for $\Phi(D)$ is densely strongly algebrable.

Theorem 4.4. Let $X$ be a separable commutative F-algebra that supports a dense freely generated subalgebra, and let $T$ be an operator on $X$. Suppose there exist a function $E: \mathbb{C} \rightarrow X$ and an entire function $\phi: \mathbb{C} \rightarrow \mathbb{C}$ with $|\phi(0)|=1$ satisfying the following:

(a) For each $\lambda \in \mathbb{C}, T E(\lambda)=\phi(\lambda) E(\lambda)$,

(b) For each $\lambda, \mu \in \mathbb{C}, E(\lambda) E(\mu)=E(\lambda+\mu)$,

(c) For each subset $\Lambda$ of $\mathbb{C}$ supporting an accumulation point in $\mathbb{C}$, the set $\{E(\lambda): \lambda \in \Lambda\}$ has dense linear span in $X$, and

(d) The integer $\min \left\{n \in \mathbb{N}: \phi^{(n)}(0) \neq 0\right\}$ is odd and $\phi$ supports an angle $\theta \in[0,2 \pi)$ and positive scalars $r, R$ so that its Phragmén-Lindelöf indicator function $h_{\phi}(\theta):=\lim \sup _{t \rightarrow \infty} \log \left|\phi\left(t e^{i \theta}\right)\right|^{\frac{1}{t}}$ satisfies

$$
\begin{cases}\left|\phi\left(t e^{i \theta}\right)\right|<1 & \text { for } 0<t<r, \text { and } \\ \left|\phi\left(R e^{i \theta}\right)\right|>\max \left\{1, e^{h_{\phi}(\theta) R}\right\} . & \end{cases}
$$

Then the generic element $f=\left(f_{n}\right)_{n=1}^{\infty}$ of $X^{\mathbb{N}}$ freely generates a dense subalgebra of $X$ consisting entirely (but zero) of hypercyclic vectors for $T$.

We postpone the technical proofs of Theorem 4.2 and Theorem 4.4 to the next subsections and see first how to derive Theorem 1.8 from them.

Proof of Theorem 1.8. Case (a) follows from Theorem 4.4 and the proof of Corollary 3.6. The remaining cases follow from Corollary 4.3. By the León-Müller Theorem we may assume in such cases that $b=0$. In Case (b) we have $\Phi(0)=1$, $\Phi^{\prime \prime}(0) \Phi(0)-\Phi^{\prime}(0)^{2}=2 a_{2}-a_{1}^{2} \neq 0$, and $\Phi^{\prime}(0)=a_{1}+a \neq 0$. For Case (c) we have $\Phi(z)=e^{z} P(z)$ where $P(z)=\prod_{n=1}^{\infty} E_{p}\left(\frac{z}{z_{n}}\right)$ with $p \in\{0,1\} . \Phi(0)=1$ and $\Phi^{\prime \prime}(0) \Phi(0) \neq \Phi^{\prime}(0)^{2}$ thanks to the assumption $\sum_{n=1}^{\infty} z_{n}^{-2} \neq 0$. So it suffices to verify that $\Phi^{\prime}(0) \neq 0$, and this holds precisely when $P^{\prime}(0)+a \neq 0$. We consider two sub-cases: If $\sum_{n=1}^{\infty}\left|z_{n}\right|^{-1}$ converges, then $p=0$ and $P^{\prime}(0)=-\sum_{n=1}^{\infty} z_{n}^{-1} \neq-a$ by our assumption. If $\sum_{n=1}^{\infty}\left|z_{n}\right|^{-1}$ diverges, then $p=1$ and $P^{\prime}(0)=0 \neq-a$ by our assumption on $a$.

4.1. Proof of Theorem 4.2, We use the following fact established with [10, Proposition 2.4, Lemma 2.10 and Lemma 3.1]:

Theorem 4.5. (B.,Papathanasiou [10]) Let $X$ be a separable commutative Falgebra that supports a dense freely generated subalgebra, and let $T$ be an operator on $X$. Suppose that for each integer $N \geq 2$ and each non-empty finite subset $A$ of $\mathbb{N}_{0}^{N}$ not containing the zero $N$-tuple there exists $\beta \in A$ satisfying:

(*) "For each non-empty open subsets $U_{1}, \ldots, U_{N}, V$ and $W$ of $X$ with $0 \in W$ there exist $f \in U_{1} \times \cdots \times U_{N}$ and $n \in \mathbb{N}$ so that $T^{n}\left(f^{\beta}\right) \in V$ and $T^{n}\left(f^{\alpha}\right) \in W$ for each $\alpha \in A \backslash\{\beta\} "$.

Then the generic element $f=\left(f_{n}\right)_{n=1}^{\infty}$ of $X^{\mathbb{N}}$ freely generates a dense subalgebra of $X$ consisting entirely (but zero) of hypercyclic vectors for $T$. 
Lemma 4.6. ([10, Lemma 3.1]) Let $A$ be a finite non-empty subset of $\mathbb{N}_{0}^{N}$, where $N \geq 2$. Then there exist positive scalars $k_{i}(i=1, \ldots, N)$ so that the functional

$$
\left(x_{i}\right)_{i=1}^{N} \mapsto \sum_{i=1}^{N} k_{i} x_{i}
$$

is injective on $A$.

Proof of Theorem 4.2. Let $N \geq 2$ and let $A$ be a non-empty finite subset of $\mathbb{N}_{0}^{N}$ not containing the zero $N$-tuple. It suffices to show there exists some $\beta$ in $A$ satisfying condition $(*)$ of Theorem 4.5. By Lemma 4.6 there exists $k \in(0, \infty)^{N}$ so that the functional

$$
\mathbb{R}^{N} \rightarrow \mathbb{R}, x \mapsto \sum_{i=1}^{N} k_{i} x_{i},
$$

is injective on $A$. Permuting the $k_{i}^{\prime} s$ if necessary, without loss of generality we may assume that the subset

$$
A_{1}:=\left\{\alpha \in A: \alpha_{1}=m\right\}
$$

of $A$ is non-empty, where $m:=\max \left\{|\alpha|_{\infty}: \alpha \in A\right\}$. Hence there exists $\beta$ in $A$ with $\beta_{1}=m$ at which the functional above attains its strict minimum over $A_{1}$. Equivalently, each $\alpha$ in $A \backslash\{\beta\}$ with $\alpha_{1}=m$ satisfies

$$
\sum_{i=2}^{N} k_{i}\left(\beta_{i}-\alpha_{i}\right)<0 .
$$

Now, let $U_{1}, \ldots, U_{N}, V$ and $W$ be non-empty open subsets of $X$ be given, with $0 \in W$. It remains to show that there exists $f$ in $U_{1} \times \cdots \times U_{N}$ and a positive integer $n$ so that

$$
\begin{aligned}
& T^{n}\left(f^{\beta}\right) \in V \\
& T^{n}\left(f^{\alpha}\right) \in W \text { for each } \alpha \in A \backslash\{\beta\} .
\end{aligned}
$$

By $(d)$, taking $w_{0}=0$ in Lemma 2.3(b) there exists some $w \in \mathbb{C} \backslash\{0\}$ so that

$$
G:[-1,1] \rightarrow \mathbb{R}, G(t)=\log |\phi(t w)|,
$$

is strictly convex and strictly increasing. In particular, since $|\phi(0)|=1$ we know that $G<0$ on $[-1,0)$ and $G>0$ on $(0,1]$. Now, let $d_{A}:=\max \left\{\sum_{i=1}^{N} \alpha_{i}: \alpha \in A\right\}$, and pick $a \in\left(0, \frac{1}{2 d_{A}}\right)$ and $b \in\left(0, \frac{a}{2 d_{A}}\right)$ and define

$$
\Lambda:=[-2 a w,-a w] \text { and } \Gamma:=[b w, 2 b w] .
$$

Then we have

$$
\sum_{k=1}^{s} \Lambda \subset\left[-d_{A} 2 a w,-a w\right] \subset \phi^{-1}(\mathbb{D}) \quad\left(1 \leq s \leq d_{A}\right)
$$

and

$$
\begin{aligned}
\sum_{k=1}^{s} \Lambda+\sum_{k=1}^{d} \Gamma & \subset\left[-d_{A} 2 a w,-a w\right]+\left[b w, d_{A} 2 b w\right] \\
& \subset\left[-d_{A} 2 a+b,-a+d_{A} 2 b\right] w \\
& \subset\left(-1,-a+d_{A} 2 b\right] w \\
& \subset(-1,0) w \subset \phi^{-1}(\mathbb{D}) \quad\left(1 \leq s<d_{A}, \text { and } 1 \leq d \leq d_{A}\right) .
\end{aligned}
$$


Now, since each of $\Lambda$ and $\Gamma$ has accumulation points in $\mathbb{C}$, condition $(c)$ ensures that there exist a positive integer $p$ and non-zero scalars $a_{i, j}, b_{j}, \lambda_{i, j}, \gamma_{j}$ with $\lambda_{i, j} \in \Lambda$, $\gamma_{j} \in \frac{1}{m} \Gamma(1 \leq i \leq N, 1 \leq j \leq p)$ so that

$$
\begin{aligned}
B & :=\sum_{j=1}^{p} b_{j} E\left(m \gamma_{j}\right) \in V \\
L_{i} & :=\sum_{j=1}^{p} a_{i, j} E\left(\lambda_{i, j}\right) \in U_{i} \quad(1 \leq i \leq N) .
\end{aligned}
$$

Moreover, we may assume that $\gamma_{1}, \ldots, \gamma_{p}$ are pairwise distinct.Next, for each positive integer $n$ let

$$
R_{n}:=\sum_{j=1}^{p} c_{j} E\left(\gamma_{j}\right)
$$

where for each $(1 \leq j \leq p)$ the scalar $c_{j}=c_{j}(n)$ is a solution of

$$
z^{m}=b_{j} \frac{n^{\sum_{i=2}^{N} k_{i} \beta_{i}}}{\phi\left(m \gamma_{j}\right)^{n}} .
$$

Notice that $c_{j}=c_{j}(n) \rightarrow 0$ as $n \rightarrow \infty$, since $\left|\phi\left(m \gamma_{j}\right)\right|>1$. So letting

$$
\left\{\begin{array}{l}
f_{1}=f_{1, n}:=L_{1}+R_{n} \\
f_{i}=f_{i, n}:=L_{i}+\frac{1}{n^{k_{i}}} \quad(2 \leq i \leq N),
\end{array}\right.
$$

we have that $f=\left(f_{1}, \ldots f_{N}\right) \in U_{1} \times \cdots \times U_{N}$ whenever $n$ is large enough. Now, for each $\alpha$ in $A$ let $\mathcal{I}_{\alpha}$ denote the set of elements $(u, v, \ell)$ in $\left(\mathbb{N}_{0}^{p}\right)^{N} \times \mathbb{N}_{0}^{p} \times \mathbb{N}_{0}^{N-1}$ for which $\left|u_{1}\right|+|v|=\alpha_{1}$ and $\left|u_{i}\right|+\ell_{i}=\alpha_{i}(2 \leq i \leq N)$. Then for each $\alpha$ in $A$ and each positive integer $n$ we have

$$
\begin{aligned}
T^{n}\left(f^{\alpha}\right) & =T^{n}\left(\prod_{i=1}^{N} f_{i}^{\alpha_{i}}\right) \\
& =\sum_{(u, v, \ell) \in \mathcal{I}_{\alpha}} X(u, v, \ell, n) E(\lambda \cdot u+\gamma \cdot v),
\end{aligned}
$$

where each $X_{\alpha}(u, v, \ell, n)$ is given by

$$
X_{\alpha}(u, v, \ell, n)=\left(\begin{array}{c}
\alpha_{1} \\
u_{1} v
\end{array}\right) \prod_{i=2}^{N}\left(\begin{array}{c}
\alpha_{i} \\
u_{i} \ell_{i}
\end{array}\right) a^{u} c^{v} \frac{(\phi(\lambda \cdot u+\gamma \cdot v))^{n}}{n^{\sum_{s=2}^{N} k_{s} \ell_{s}}}
$$

where

$$
\begin{aligned}
\lambda \cdot u+\gamma \cdot v & =\sum_{i=1}^{N} \sum_{j=1}^{p} \lambda_{i, j} u_{i, j}+\sum_{j=1}^{p} \gamma_{j} v_{j}, \\
a^{u} & =\prod_{1 \leq i \leq N, 1 \leq j \leq p} a_{i, j}^{u_{i, j}}, \text { and } \\
c^{v} & =\prod_{j=1}^{p} c_{j}^{v_{j}} .
\end{aligned}
$$

So it suffices to show that for each $\alpha \in A \backslash\{\beta\}$

$$
X_{\alpha}(u, v, \ell, n) \underset{n \rightarrow \infty}{\rightarrow} 0 \quad\left((u, v, \ell) \in \mathcal{I}_{\alpha}\right)
$$


and that for each $(u, v, \ell) \in \mathcal{I}_{\beta}$

$$
\lim _{n \rightarrow \infty} X_{\beta}(u, v, \ell, n)= \begin{cases}b_{j} & \text { if }(u, v) \in\left\{\left(0, m e_{j}\right): j=1, \ldots, p\right\} \\ 0 & \text { otherwise }\end{cases}
$$

where $\left\{e_{1}, \ldots, e_{p}\right\}$ is the standard basis of $\mathbb{C}^{p}$. Now, let $\alpha \in A$ and let $(u, v, \ell) \in \mathcal{I}_{\alpha}$ be given. Notice that by (4.4) and (4.5) we have

$$
\left|X_{\alpha}(u, v, \ell, n)\right| \leq(\text { Constant }) n^{\sum_{s=2}^{N}\left(\frac{|v|}{m} k_{s} \beta_{s}-k_{s} \ell_{s}\right)} \Theta_{u, v}^{n},
$$

where

$$
\Theta_{u, v}=\frac{|\phi(\lambda \cdot u+\gamma \cdot v)|}{\prod_{j=1}^{p}\left|\phi\left(m \gamma_{j}\right)\right|^{\frac{v_{j}}{m}}}
$$

We consider four cases.

Case 1: $1 \leq|u|<d_{A}$. By (4.2) and (4.3) we have

$$
\lambda \cdot u+\gamma \cdot v \in \sum_{k=1}^{|u|} \Lambda+\sum_{k=1}^{|v|} \Gamma \subset \phi^{-1}(\mathbb{D})
$$

Since $\left|\phi\left(m \gamma_{j}\right)\right|>1$ for each $1 \leq j \leq p$, we have $\Theta_{u, v} \in[0,1)$ and by (4.8) we have $X_{\alpha}(u, v, \ell, n) \underset{n \rightarrow \infty}{\rightarrow} 0$.

Case 2: $|u|=d_{A}$. So $|v|=0$ in this case, and

$$
\lambda \cdot u+\gamma \cdot v=\gamma \cdot v \in \sum_{k=1}^{d_{A}} \Lambda \in \phi^{-1}(\mathbb{D})
$$

by (4.2), and arguing as in Case 1 we have $X_{\alpha}(u, v, \ell, n) \underset{n \rightarrow \infty}{\rightarrow} 0$.

Case 3: $|u|=0$ and $|v|_{\infty}<m$. In this case we have $u_{1}=\cdots=u_{N}=0$, and hence $|v|=\alpha_{1}<m$ and $\ell_{i}=\alpha_{i}$ for each $2 \leq i \leq N$. Now, notice that

$$
\gamma \cdot v=0 \frac{m-|v|}{m}+m \gamma_{1} \frac{v_{1}}{m}+\cdots+m \gamma_{p} \frac{v_{p}}{m}
$$

is a non-trivial convex combination of the points $0, m \gamma_{1}, \ldots, m \gamma_{p}$ in $[0, w]$. Since $|\phi(0)|=1$, we have

$$
\Theta_{u, v}=\frac{|\phi(\gamma \cdot v)|}{|\phi(0)|^{\frac{m-|v|}{m}} \prod_{j=1}^{p}\left|\phi\left(m \gamma_{j}\right)\right|^{\frac{v_{j}}{m}}}<1
$$

thanks to the strict convexity of $t \mapsto \log |\phi(t w)|$ on $[-1,1]$, and again by (4.8) we have $X_{\alpha}(u, v, \ell, n) \underset{n \rightarrow \infty}{\rightarrow} 0$.

Case 4: $|u|=0$ and $|v|_{\infty}=m$. Suppose first that $v \notin\left\{m e_{1}, \ldots, m e_{p}\right\}$. Then $\lambda \cdot u+\gamma \cdot v=\gamma \cdot v$ is a nontrivial convex combination of the points $m \gamma_{1}, \ldots, m \gamma_{p}$ in $[0, w]$, and again $\Theta_{u, v} \in[0,1)$ thanks to the strict convexity of the map $t \mapsto$ $\log |\phi(t w)|$ on $[0,1]$, so $X_{\alpha}(u, v, \ell, n) \underset{n \rightarrow \infty}{\rightarrow} 0$ by (4.8).

Finally, suppose $v=m e_{j}$ for some fixed $j \in\{1, \ldots, p\}$. Here we have $\Theta_{u, v}=1$, $\alpha_{1}=m$, and $\ell_{i}=\alpha_{i}$ for each $i \in\{2, \ldots, N\}$, as $u_{1}=\cdots=u_{N}=0$. So by (4.4) 
and (4.5) we have

$$
\begin{aligned}
X_{\alpha}(u, v, \ell, n) & =\left(\begin{array}{c}
\alpha_{1} \\
u_{1} v
\end{array}\right) \prod_{i=2}^{N}\left(\begin{array}{c}
\alpha_{i} \\
u_{i} \ell_{i}
\end{array}\right) a^{u} c^{v} \frac{(\phi(\lambda \cdot u+\gamma \cdot v))^{n}}{n^{\sum_{s=2}^{N} k_{s} \ell_{s}}} \\
& =c_{j}^{m} \frac{\phi\left(m \gamma_{j}\right)^{n}}{n^{\sum_{s=2}^{N} k_{s} \alpha_{s}}} \\
& =b_{j} n^{\sum_{i=2}^{N}\left(k_{i} \beta_{i}-k_{i} \alpha_{i}\right)} .
\end{aligned}
$$

So if $\alpha \neq \beta$ we have

$$
X_{\alpha}(u, v, \ell, n)=b_{j} n^{\sum_{i=2}^{N}\left(k_{i} \beta_{i}-k_{i} \alpha_{i}\right)} \underset{n \rightarrow \infty}{\rightarrow} 0
$$

thanks to (4.1), while if $\alpha=\beta$ we have

$$
X_{\alpha}(u, v, \ell, n)=X_{\beta}\left(0, m e_{j}, \ell, n\right)=b_{j} \underset{n \rightarrow \infty}{\rightarrow} b_{j} .
$$

That is, (4.6) and (4.7) are satisfied, and the proof is now complete.

\subsection{Proof of Theorem 4.4.}

Proof of Theorem 4.4. Let $N \geq 2$ and let $A$ be a non-empty finite subset of $\mathbb{N}_{0}^{N}$ not containing the zero $N$-tuple. As in the proof of Theorem 4.2, we may assume that the subset

$$
A_{1}:=\left\{\alpha \in A: \alpha_{1}=m\right\}
$$

of $A$ is non-empty, where $m:=\max \left\{|\alpha|_{\infty}: \alpha \in A\right\}$, and that there exist $k \in$ $(0, \infty)^{N}$ and $\beta$ in $A_{1}$ so that

$$
\sum_{i=2}^{N} k_{i}\left(\beta_{i}-\alpha_{i}\right)<0
$$

for each $\alpha \in A_{1}$. Now, let $U_{1}, \ldots, U_{N}, V$ and $W$ be non-empty open subsets of $X$ be given, with $0 \in W$. By Theorem [4.5. it suffices to show that there exist $f$ in $U_{1} \times \cdots \times U_{N}$ and a positive integer $n$ so that

$$
\begin{aligned}
& T^{n}\left(f^{\beta}\right) \in V \\
& T^{n}\left(f^{\alpha}\right) \in W \text { for each } \alpha \in A \backslash\{\beta\} .
\end{aligned}
$$

By (d) and Remark 1.7 as in the proof of Theorem 1.6 we may get $w_{0}, z_{0} \in \mathbb{C}$ with $w_{0} \in\left\{t z_{0}: t>1\right\}$ so that

(i) $\left|\phi\left(w_{0}\right)\right|>1$ and $|\phi|<1$ on $\left[-z_{0}, 0\right) \cup\left(0, z_{0}\right]$,

(ii) $\left|\phi\left(d w_{0}\right)\right|<\left|\phi\left(w_{0}\right)\right|^{d}$ for each $d \in\{2, \ldots, m\}$, and

(iii) The map $t \mapsto\left|\phi\left(w_{0}+t z_{0}\right)\right|$ is strictly increasing on $(-1,1)$.

Let $\gamma_{1} \in\left(0, \frac{z_{0}}{m}\right) \cap D(0,1)$ close enough to zero so that

(i') $1<\left|\phi\left(w_{0}+(m-1) \gamma_{1}\right)\right|$,

(ii') for each $d \in\{2, \ldots, m\}$ and $s \in\{0, \ldots, m-2\}$

$$
\left|\phi\left(d w_{0}+s \gamma_{1}\right)\right|^{\frac{1}{d}}<\left|\phi\left(w_{0}+(m-1) \gamma_{1}\right)\right|, \quad \text { and }
$$

(iii') the function $t \mapsto\left|\phi\left(w_{0}+t \gamma_{1}\right)\right|$ is strictly increasing on the interval $\left(-d_{A}-1, d_{A}+1\right)$, where $d_{A}=\max _{\alpha \in A}|\alpha|$.

By $\left(i^{\prime}\right)$ and $\left(i i^{\prime}\right)$, there exists $\delta \in(0,1)$ small enough so that

(1) $1<\left|\phi\left(\lambda+(m-1) \gamma_{1}\right)\right|$ for each $\lambda \in D\left(w_{0}, \delta\right)$, and 
(2) for each $\lambda, \lambda_{1}^{\prime}, \ldots, \lambda_{d}^{\prime}$ in $D\left(w_{0}, \delta\right)$ and $z$ in $D(0, \delta)$ and each $(d, s) \in\{2, \ldots, m\} \times\{0, \ldots, m-2\}$,

$$
\left|\phi\left(\lambda_{1}^{\prime}+\cdots+\lambda_{d}^{\prime}+s \gamma_{1}+z\right)\right|^{\frac{1}{d}}<\left|\phi\left(\lambda+(m-1) \gamma_{1}\right)\right| .
$$

Reducing $\delta>0$ if necessary, by $\left(i i i^{\prime}\right)$ we may further assume that

(3) for each $\lambda$ in $\left(w_{0}-\delta \gamma_{1}, w_{0}+\delta \gamma_{1}\right)$, the function

$$
t \mapsto\left|\phi\left(\lambda+t \gamma_{1}\right)\right|
$$

is strictly increasing on $\left[-d_{A}, d_{A}\right]$.

Let $a_{1,1}:=1, \gamma_{1,1}:=\gamma_{1}$, and

$$
R>\frac{d_{A}}{\delta}
$$

By (c) there exist $b_{1}, \ldots, b_{p}, a_{1,2}, \ldots, a_{1, p} \in \mathbb{C} \backslash\{0\}$, pairwise distinct $\lambda_{1}, \ldots, \lambda_{p} \in$ $\left(w_{0}-\delta \gamma_{1}, w_{0}+\delta \gamma_{1}\right)$, and

$$
\gamma_{1,2}, \ldots, \gamma_{1, p} \in\left(0, \frac{\gamma_{1}}{R}\right)
$$

so that

$$
\begin{aligned}
B & :=\sum_{j=1}^{p} b_{j} E\left(\lambda_{j}+(m-1) \gamma_{1}\right) \in V \\
L_{1} & :=\sum_{j=1}^{p} a_{1, j} E\left(\gamma_{1, j}\right) \in U_{1} .
\end{aligned}
$$

Now, let $\eta:=\min _{1 \leq j \leq p}\left|\gamma_{1, j}\right|$. Again by $(c)$, there exist an integer $q \geq p$ and non-zero scalars $a_{i, j}, \gamma_{i, j}((i, j) \in\{2, \ldots, N\} \times\{1, \ldots, q\})$ with

$$
\gamma_{i, j} \in\left[-z_{0}, 0\right) \cap D\left(0, \frac{\eta}{R}\right)
$$

so that

$$
L_{i}:=\sum_{j=1}^{p} a_{i, j} E\left(\gamma_{i, j}\right) \in U_{i} \quad(2 \leq i \leq N) .
$$

Enlarging $p$ if necessary (and choosing some new $\gamma_{1, j}$ 's in $\left(\eta \frac{\gamma_{1}}{\left|\gamma_{1}\right|}, \frac{\gamma_{1}}{R}\right)$ and corresponding small $a_{1, j}$ 's in $\mathbb{C} \backslash\{0\}$ so that (4.13) is preserved), we may assume that $q=p$. Next, for each positive integer $n$ let

$$
R_{n}:=\sum_{j=1}^{p} c_{j} E\left(\lambda_{j}\right)
$$

where for each $j \in\{1, \ldots, p\}$ the scalar $c_{j}=c_{j}(n)$ is a solution of

$$
b_{j}=m c_{j} \frac{n^{\sum_{i=2}^{N} k_{i} \beta_{i}}}{\phi\left(\lambda_{j}+(m-1) \gamma_{1}\right)^{n}} .
$$

Notice that $c_{j}=c_{j}(n) \rightarrow 0$ as $n \rightarrow \infty$, as $\left|\phi\left(\lambda_{j}+(m-1) \gamma_{1}\right)\right|>1$ by (1). So letting

$$
\left\{\begin{array}{l}
f_{1}=f_{1, n}:=L_{1}+R_{n} \\
f_{i}=f_{i, n}:=L_{i}+\frac{1}{n^{k_{i}}} \quad(2 \leq i \leq N),
\end{array}\right.
$$

we have that $f=\left(f_{1}, \ldots f_{N}\right) \in U_{1} \times \cdots \times U_{N}$ whenever $n$ is large enough. Again, for each $\alpha$ in $A$ let $\mathcal{I}_{\alpha}$ denote the set of elements $(u, v, \ell)$ in $\left(\mathbb{N}_{0}^{p}\right)^{N} \times \mathbb{N}_{0}^{p} \times \mathbb{N}_{0}^{N-1}$ 
for which $\left|u_{1}\right|+|v|=\alpha_{1}$ and $\left|u_{i}\right|+\ell_{i}=\alpha_{i}(2 \leq i \leq N)$. Then for each $\alpha$ in $A$ and each positive integer $n$ we have

$$
\begin{aligned}
T^{n}\left(f^{\alpha}\right) & =T^{n}\left(\prod_{i=1}^{N} f_{i}^{\alpha_{i}}\right) \\
& =\sum_{(u, v, \ell) \in \mathcal{I}_{\alpha}} X_{\alpha}(u, v, \ell, n) E(\lambda \cdot u+\gamma \cdot v),
\end{aligned}
$$

where

$$
\begin{aligned}
X_{\alpha}(u, v, \ell, n) & =\left(\begin{array}{c}
\alpha_{1} \\
u_{1} v
\end{array}\right) \prod_{i=2}^{N}\left(\begin{array}{c}
\alpha_{i} \\
u_{i} \ell_{i}
\end{array}\right) a^{u} c^{v} \frac{(\phi(\gamma \cdot u+\lambda \cdot v))^{n}}{n^{\sum_{s=2}^{N} k_{s} \ell_{s}}} \\
\lambda \cdot u+\gamma \cdot v & =\sum_{i=1}^{N} \sum_{j=1}^{p} \lambda_{i, j} u_{i, j}+\sum_{j=1}^{p} \gamma_{j} v_{j}, \\
a^{u} & =\prod_{1 \leq i \leq N, 1 \leq j \leq p} a_{i, j}^{u_{i, j}}, \text { and } \\
c^{v} & =\prod_{j=1}^{p} c_{j}^{v_{j}} .
\end{aligned}
$$

Notice that for each $k \in\{1, \ldots, p\}$ the element $(u, v, \ell) \in \mathcal{I}_{\beta}$ given by $v=e_{k}$, $u_{1}=(m-1) e_{1},\left|u_{2}\right|=\cdots=\left|u_{N}\right|=0$, and $\ell=\left(\beta_{i}\right)_{i=2}^{N}$ satisfies

$$
\left\{\begin{array}{l}
X_{\beta}(u, v, \ell)=b_{k} \text { and } \\
E(\gamma \cdot u+\lambda \cdot v)=E\left(\lambda_{k}+(m-1) \gamma_{1}\right) .
\end{array}\right.
$$

Hence by (4.15) and (4.17) it suffices to show that

$$
X_{\alpha}(u, v, \ell, n) \underset{n \rightarrow \infty}{\rightarrow} 0
$$

except for the $p$ elements $(u, v, \ell)$ of $\cup_{\alpha \in A} \mathcal{I}_{\alpha}$ considered in (4.19). To this end, let $\alpha \in A$ and let $(u, v, \ell) \in \mathcal{I}_{\alpha}$ be fixed, not of the form considered in (4.19). Notice that

$$
\left|X_{\alpha}(u, v, \ell, n)\right| \leq K n^{\sum_{s=2}^{N}\left(\frac{|v|}{m} k_{s} \beta_{s}-k_{s} \ell_{s}\right)} \Theta_{u, v}^{n}
$$

where

$$
\Theta_{u, v}=\frac{|\phi(\gamma \cdot u+\lambda \cdot v)|}{\prod_{j=1}^{p}\left|\phi\left(\lambda_{j}+(m-1) \gamma_{1}\right)\right|^{\frac{v_{j}}{m}}}
$$

and where $K$ is a constant that does not depend on $n$. In particular, (4.20) holds whenever $\Theta_{u, v}<1$. We consider the following cases:

Case 1: $|v|=0$. So $\left|u_{1}\right|=\alpha_{1}-|v|=\alpha_{1}$ in this case. If $|u|=0$, then $\alpha_{1}=0$ and $\ell=\left(\alpha_{i}\right)_{i=2}^{N}$, and since $A$ does not contain the zero N-tuple we must have $\ell_{i}=\alpha_{i}>0$ for some $i \in\{2, \ldots, N\}$. Since $|\Phi(u \cdot \gamma)|=|\phi(0)| \leq 1$, by (4.21) and (4.9) we have

$$
\left|X_{\alpha}(u, v, \ell, n)\right|=K n^{-\sum_{s=2}^{N} \ell_{s} k_{s}}|\phi(u \cdot \gamma)|^{n} \underset{n \rightarrow \infty}{\rightarrow} 0 .
$$


So we assume $|u| \neq 0$. If $\left|u_{1}\right| \neq 0$, then by (4.11), (4.12) and $(i)$

$$
\begin{aligned}
u \cdot \gamma & =u_{1,1} \gamma_{1}+\sum_{j=2}^{p} u_{1, j} \gamma_{1, j}+\sum_{i=2}^{N} \sum_{j=1}^{p} u_{i, j} \gamma_{i, j} \\
& \in\left(0, \frac{|u|-u_{1,1}}{R}+u_{1,1}\right) \gamma_{1} \subset\left(0, z_{0}\right] \subset \phi^{-1}(\mathbb{D}),
\end{aligned}
$$

so $\Theta_{u, v}=|\phi(u \cdot \gamma)|<1$. On the other hand, if $\left|u_{1}\right|=0$, we have $\left|u_{2}\right|+\cdots+\left|u_{N}\right|=$ $|u| \neq 0$ and thus by (4.11), (4.14) and $(i)$ we have $u \cdot \gamma \in\left[-z_{0}, 0\right) \subset \phi^{-1}(\mathbb{D})$ and hence $\Theta_{u, v}=|\phi(u \cdot \gamma)|<1$.

Case 2: $|v| \in\{2, \ldots, m\}$. Here we have $u_{1,1} \leq\left|u_{1}\right|=\alpha_{1}-|v| \leq m-2$. Also, $z:=u \cdot \gamma-u_{1,1} \gamma_{1}$ has size

$$
|z|=\left|\sum_{j=2}^{p} u_{1, j} \gamma_{1, j}+\sum_{i=2}^{N} \sum_{j=1}^{p} u_{i, j} \gamma_{i, j}\right| \leq \frac{|\alpha|-|v|}{R}\left|\gamma_{1}\right| \leq \frac{d_{A}-2}{R}\left|\gamma_{1}\right|<\delta .
$$

by (4.11). So by (2) we have

$$
\begin{aligned}
|\phi(u \cdot \gamma+\lambda \cdot v)| & =\left|\phi\left(u_{1,1} \gamma_{1}+z+v \cdot \lambda\right)\right| \\
& <\left|\phi\left(\lambda_{j}+(m-1) \gamma_{1}\right)\right|^{p}(1 \leq j \leq p),
\end{aligned}
$$

and thus

$$
\Theta_{u, v}=\frac{|\phi(u \cdot \gamma+\lambda \cdot v)|}{\prod_{j=1}^{p}\left|\phi\left(\lambda_{j}+(m-1) \gamma_{1}\right)\right|^{v_{j}}}=\prod_{j=1}^{p} \frac{|\phi(u \cdot \gamma+\lambda \cdot v)|^{\frac{v_{j}}{d}}}{\left|\phi\left(\lambda_{j}+(m-1) \gamma_{1}\right)\right|^{v_{j}}}<1
$$

Case 3: $|v|=1$. So $v=e_{k}$ for some $k \in\{1, \ldots, p\}$, and $0 \leq u_{1,1} \leq\left|u_{1}\right|=\alpha_{1}-1$. If $\left|u_{1}\right|=0$, then by (4.14) we have $u \cdot \gamma=-t \gamma_{1}$ for some $t \in[0, m-1)$. Hence by $(3)$

$$
|\phi(u \cdot \gamma+v \cdot \lambda)|<\left|\phi\left(\lambda_{k}+(m-1) \gamma_{1}\right)\right|
$$

giving $\Theta_{u, v}<1$. So assume that $\left|u_{1}\right|>0$. Now, if $u_{1,1}<m-1$, then by (4.11) and (4.12)

$$
u \cdot \gamma+v \cdot \lambda=\lambda_{k}+t \gamma_{1}
$$

for some $t \in(0, m-1)$, giving again by $(3)$

$$
|\phi(u \cdot \gamma+v \cdot \lambda)|<\left|\phi\left(\lambda_{k}+(m-1) \gamma_{1}\right)\right|
$$

and thus $\Theta_{u, v}<1$. Finally, if $u_{1,1}=m-1$, then we must have $\alpha_{1}=m$ and $u_{1}=(m-1) e_{1}$. Here we have two possibilities: either $\left|u_{i}\right|>0$ for some $2 \leq i \leq N$ or else $\left|u_{2}\right|=\cdots=\left|u_{N}\right|=0$. In the former case we have by (4.11) and (4.12)

$$
u \cdot \gamma+v \cdot \lambda=\lambda_{k}+t \gamma_{1}
$$

for some $t \in(0, m-1)$, giving $\Theta_{u, v}<1$. In the latter case we have $\ell=\left(\alpha_{i}\right)_{i=2}^{N}$ and $u \cdot \gamma+v \cdot \lambda=\lambda_{k}+(m-1) \gamma_{1}$, giving $\Theta_{u, v}=1$. Since $(u, v, \ell)$ is not of the form considered in (4.19), then $\alpha \neq \beta$. Hence by (4.21) and (4.9) we have

$$
\left|X_{\alpha}(u, v, \ell, n)\right| \leq K n^{\sum_{s=2}^{N}\left(k_{s} \beta_{s}-k_{s} \alpha_{s}\right)} \underset{n \rightarrow \infty}{\rightarrow} 0
$$

and 4.20) holds. 


\section{REFERENCES}

[1] R. M. Aron et al., Lineability: the search for linearity in mathematics, Monographs and Research Notes in Mathematics, CRC Press, Boca Raton, FL, 2016. MR3445906

[2] R. M. Aron et al., Powers of hypercyclic functions for some classical hypercyclic operators, Integral Equations Operator Theory 58 (2007), no. 4, 591-596. MR2329137

[3] F. Bayart, Hypercyclic algebras, J. Funct. Anal. 276 (2019), no. 11, 3441-3467. MR3944300

[4] F. Bayart and Matheron, Dynamics of linear operators, Cambridge Tracts in Mathematics, 179, Cambridge University Press, Cambridge, 2009. MR2533318

[5] A. Bartoszewicz and S. Głąb, Strong algebrability of sets of sequences and functions, Proc. Amer. Math. Soc. 141 (2013), no. 3, 827-835. MR3003676

[6] L. Bernal-González and M. C. Calderón-Moreno, Hypercyclic algebras for D-multiples of convolution operators, Proc. Amer. Math. Soc. 147 (2019), no. 2, 647-653. MR3894904

[7] L. Bernal-González, D. Pellegrino and J. B. Seoane-Sepúlveda, Linear subsets of nonlinear sets in topological vector spaces, Bull. Amer. Math. Soc. (N.S.) 51 (2014), no. 1, 71-130. MR3119823

[8] J. Bès, J. A. Conejero and D. Papathanasiou, Convolution operators supporting hypercyclic algebras, J. Math. Anal. Appl. 445 (2017), no. 2, 1232-1238. MR3545235

[9] J. Bès, J. A. Conejero and D. Papathanasiou, Hypercyclic algebras for convolution and composition operators, J. Funct. Anal. 274 (2018), no. 10, 2884-2905. MR3777634

[10] J. Bès and D. Papathanasiou, Algebrable sets of hypercyclic vectors for convolution operators. arXiv e-prints, 2017.

[11] R. P. Boas, Jr., Entire functions, Academic Press Inc., New York, 1954. MR0068627

[12] T. W. Gamelin, Complex analysis, Undergraduate Texts in Mathematics, Springer-Verlag, New York, 2001. MR1830078

[13] G. Godefroy and J. H. Shapiro, Operators with dense, invariant, cyclic vector manifolds, J. Funct. Anal. 98 (1991), no. 2, 229-269. MR1111569

[14] K.-G. Grosse-Erdmann and J. Falcó. Algebrability of the set of hypercyclic vectors for backward shift operators. arXiv:1807.04544 v1, 2018.

[15] K.-G. Grosse-Erdmann and A. Peris Manguillot, Linear chaos, Universitext, Springer, London, 2011. MR2919812

[16] F. León-Saavedra and V. Müller, Rotations of hypercyclic and supercyclic operators, Integral Equations Operator Theory 50 (2004), no. 3, 385-391. MR2104261

[17] S. Shkarin, On the set of hypercyclic vectors for the differentiation operator, Israel J. Math. 180 (2010), 271-283. MR2735066

[18] J. Wengenroth, Hypercyclic operators on non-locally convex spaces, Proc. Amer. Math. Soc. 131 (2003), no. 6, 1759-1761. MR1955262

J. Bès, Department of Mathematics and Statistics, Bowling Green State UniverSity, Bowling Green, Ohio 43403, USA.

E-mail address: jbes@bgsu.edu

R. Ernst, lmpa, Centre Universitaire de la Mi-Voix, Maison de la Recherche Blaise-Pascal, 50 Rue Ferdinand Buisson, BP 699, 62228 Calais Cedex, France.

E-mail address: romuald.ernst@math.cnrs.fr

A. Prieto, Departamento de Análisis Matemático y Matemática Aplicada, Universidad Complutense de Madrid, Plaza de Ciencias 3, 28040 Madrid, Spain

E-mail address: angelin@mat.ucm.es 